\title{
Reheating-volume measure in the landscape
}

\author{
Sergei Winitzki \\ Department of Physics, Ludwig-Maximilians University, Munich, Germany
}

\begin{abstract}
I recently proposed the "reheating-volume" (RV) prescription as a possible solution to the measure problem in "multiverse" cosmology. The goal of this work is to extend the RV measure to scenarios involving bubble nucleation, such as the string theory landscape. In the spirit of the RV prescription, I propose to calculate the distribution of observable quantities in a landscape that is conditioned in probability to nucleate a finite total number of bubbles to the future of an initial bubble. A general formula for the relative number of bubbles of different types can be derived. I show that the RV measure is well-defined and independent of the choice of the initial bubble type, as long as that type supports further bubble nucleation. Applying the RV measure to a generic landscape, I find that the abundance of Boltzmann brains is always negligibly small compared with the abundance of ordinary observers in the bubbles of the same type. As an illustration, I present explicit results for a toy landscape containing four vacuum states and for landscapes with a single high-energy vacuum and a large number of low-energy vacua.
\end{abstract}

\section{INTRODUCTION AND SUMMARY}

In many cosmological scenarios the fundamental theory does not predict with certainty the values of observable cosmological parameters, such as the effective cosmological constant and the masses of elementary particles. This is the case even for some models of inflation driven by a scalar field (see e.g. 1, 2] for early work) as well as for the "landscape of string theory" [3, 4, 5, 5], see also the "recycling universe" 7] models. In these latter models, the fundamental theory admits a large number of disjoint vacuum states. Transitions between these states are possible through bubble nucleation; the interior of a bubble appears as an infinite homogeneous open universe [8], if one disregards the small probability of bubble collisions (see Refs. [9, 10] for analyses of bubble collisions). The presently observed universe is situated within a bubble (called a "pocket universe") of some type.

A common feature of these cosmological models is the presence of eternal inflation, i.e. the absence of a global end to inflation in the entire spacetime (see Refs. [11, 12, 13] for reviews). Eternal inflation gives rise to infinitely many causally disconnected regions of the spacetime where the cosmological observables may have significantly different values. In the context of the stringtheoretic landscape, eternal inflation entails the nucleation of (potentially) infinitely many nested bubbles of different vacuum types.

The program outlined in the early works [14, 15, 16], which dealt with eternal inflation of random walk type, was to calculate the probability distribution of the cosmological parameters as measured by an observer randomly located in the spacetime. The main diffuculty in obtaining such probability distributions is due to the infinite volume of regions where an observer may be located. An eternally inflating universe contains an infinite, inhomogeneous, and topologically complicated spacelike hypersurface (the reheating surface) where observers may be expected to appear with a constant density per unit 3 -volume.
In the landscape scenarios, one encounters a kind of infinity that is in some sense more ill-behaved than in the random-walk inflationary scenarios. Not only each pocket universe may contain infinitely many observers, but also the number of different pocket universes in the entire spacetime is infinite. Pocket universes of different types are not statistically equivalent to each other because they have different rates of nucleation of other pocket universes. There seems to be no natural ordering on the set of all pocket universes throughout the spacetime, since most of the pocket universes are spacelike separated. To emphasize the mutual causal independence of pocket universes, one calls such a spacetime a "multiverse."

In summary, eternal inflation is a stochastic process that generates a topologically complicated and noncompact locus of points where observers may appear. A "random location" of an observer within that locus is a mathematically undefined concept, similarly to the concept of an integer number "uniformly chosen" among all the integers, or a real number "uniformly chosen" among all the reals. This is the root cause of the technical and conceptual difficulties known collectively as the "measure problem" in multiverse cosmology (see Refs. [12, 13, 17, 18, 19, 20] for reviews). Nevertheless, one may try to formulate a prescription for calculating probabilities of observer-based events. Such a prescription, also called a "measure," should in some sense correspond to the intuitive notion of probability of observation at a "random" location in the spacetime.

Several measure prescriptions have been proposed in the literature. The proposals that apply directly to landscape scenarios are the "holographic" measure [21, 22. (see also the recent proposal [23]), the "comoving horizon cutoff" [24, 25, 26], the "stationary measure" [20, 27], the measure on transitions 28], and the "pseudo-comoving" measure [29, 30, 31, 32]. In the absence of a unique definition of the measure, one judges a cutoff prescription viable if its predictions are not obviously pathological. Possible pathologies include the dependence on choice of spacetime coordinates [33, 34], the "young- 
ness paradox" 35, 36, and the "Boltzmann brain" problem 29, 37, 38, 39, 40, 41, 42, 43. . Various measures have been used for predicting cosmological parameters, most notably the cosmological constant, in the landscape scenarios (see, e.g., Refs. [27, 44, 45, 46, 47, 48, 49]).

The purpose of this paper is to extend the most recently proposed "reheating-volume" (RV) measure [50, 51], originally formulated in the context of random-walk inflation, to landscape scenarios. The basic idea of the RV proposal is to select multiverses that are very large but (by rare chance) have a finite total number of observers. In the context of a string landscape scenario (or a "recycling universe"), this can happen if sufficiently many anti-de Sitter or Minkowski bubbles nucleate everywhere, collide, and merge. In such a case, there will be a finite time after which no de Sitter regions remain and no further nucleations can occur. Hence, there will be a finite time after which no more observers are created anywhere. By this construction, one obtains a subensemble of multiverses having a fixed, finite total number $N_{\text {obs }}$ of observers. These finite multiverses with very large $N_{\text {obs }}$ are regarded as controlled approximations to the actual infinite multiverse. The observer-weighted statistical distribution of any quantity within a finite multiverse can be obtained by ordinary counting, since the total number of observers within any such multiverse is finite. The limit of that statistical distribution as $N_{\text {obs }} \rightarrow \infty$ is the final result of the RV prescription.

It was shown in Refs. [50, 51] that the RV measure is gauge-invariant, independent of the initial conditions, and free of the youngness paradox in the context of random-walk inflation. Presently I investigate whether the same features persist in an application of the RV measure to landscape scenarios. In particular, it is important to obtain RV predictions with respect to the "Boltzmann brain" problem that has been widely discussed.

In principle, the RV prescription can be extended to landscape models in different ways, depending on the precise choice of the ensemble of finite multiverses. The ensemble of multiverses with a fixed total number of observers $N_{\text {obs }}$ (where one counts both the ordinary observers and the "Boltzmann brains") appears to be the natural choice. However, it is difficult to compute the number of observers directly and unambiguously. Instead of the total number of observers, I propose to fix the total number $n_{\text {tot }}$ of bubbles nucleated to the future of an initial bubble.

The total number of ordinary observers in bubbles of a given kind is proportional to the volume of the reheating surface in those bubbles. It is known that the "square bubble" approximation [42], which neglects the effects of bubble wall geometry, is adequate for the purposes of volume counting. Then the evolution of the landscape is well described by the approximate model called "inflation in a box" [50, 52, 53]. In that approximation, one keeps track only of the number of new bubbles nucleated in previously existing bubbles, and each new bubble is assumed to be instantaneously nucleated exactly of Hubble size in comoving coordinates. Motivated by this approximation, in this paper I study a simplified definition of the RV measure for a landscape scenario (see Sec. IIfor details): One requires the total number of bubbles of all types, $n_{\text {tot }}$, to be finite and evaluates the statistical distribution of bubble types (or other cosmological observables) in the limit $n_{\text {tot }} \rightarrow \infty$. In principle, this limit can be calculated if the bubble nucleation rates are known. Since this paper is a first attempt to perform this techically challenging calculation, I concentrate only on bubble abundances and on the relative abundance of Boltzmann brains. I neglect the increased number of observers due to additional slowroll inflation within bubbles; this effect was considered in Ref. [50] and requires additional complications in the formalism.

A landscape scenario may be specified by enumerating the available $N$ types of vacua by a label $j(j=1, \ldots, N)$ and by giving the Hubble rates $H_{j}$ within bubbles of type $j$. One can, in principle, compute the nucleation rate $\Gamma_{j \rightarrow k}$ describing the probability (per unit four-volume of spacetime) of creating a bubble of type $k$ within bubbles of type $j .{ }^{1}$ It is convenient to work with the dimensionless rates,

$$
\kappa_{j \rightarrow k} \equiv \frac{4 \pi}{3} \Gamma_{j \rightarrow k} H_{j}^{-4} .
$$

The rate $\kappa_{j \rightarrow k}$ equals the probability of having a bubble of type $k$ within a 3 -volume of one horizon in a bubble of type $j$, during a single Hubble time. ${ }^{2}$ Explicit expressions for $\kappa_{j \rightarrow k}$ are available in some landscape scenarios. In what follows, I assume that $\kappa_{j \rightarrow k}$ are known.

In Sec. III I apply the RV measure proposal to a toy model of the landscape with four vacua (the FABI model of Ref. 24]). In this model, the vacua labeled $F$ and $I$ are de Sitter (dS) and the vacua labeled $A$ and $B$ are anti-de Sitter (AdS) states. One assumes that only the transitions $F \rightarrow I, I \rightarrow F, F \rightarrow A$, and $I \rightarrow B$ are allowed, with known nucleation rates $\kappa_{F I}, \kappa_{I F}$, etc., per unit Hubble 4-volume. I show in Eq. (63) that the RVregulated bubble abundances depend on the value of the dimensionless number

$$
\eta \equiv\left(\frac{\kappa_{I B}}{\kappa_{F A}}\right)^{\nu+1} \frac{\kappa_{F I}}{\kappa_{I F}}, \quad \nu \equiv e^{3} .
$$

Here the constant $\nu$, introduced for convenience, is simply the number of statistically independent Hubble regions after one $e$-folding. Barring fine-tuned cases, one expects that the value of $\eta$ is either much larger than 1 or much smaller than 1, since the nucleation rates may differ by exponentially many orders of magnitude. By relabeling the vacua $(F \leftrightarrow I$ and $A \leftrightarrow B)$ if necessary, we may

\footnotetext{
${ }^{1}$ For some recent work concerning the determination of the bubble nucleation rates, see Refs. [54, [55, [56, [57, 58].

2 The notation $\kappa_{j \rightarrow k}$, chosen here for its visual clarity, corresponds to $\kappa_{k j}$ of Ref. [24] and to $\Gamma_{j k}$ of Ref. [50].
} 
assume that $\eta \ll e^{-6}$. Then the bubble abundances are approximately described by the ratios

$$
p(I): p(F): p(A): p(B) \approx \frac{1}{\nu^{2}}: \frac{1}{\nu}: 1:\left[\frac{\eta}{\nu^{\nu-1}}\right]^{\frac{1}{\nu+1}} .
$$

This result can be interpreted as follows. Each of the $I$ bubbles produces $\nu$ bubbles of type $F$, and each of the $F$ bubbles produces $\nu$ bubbles of type $A$. The abundance of $B$ bubbles is neligible compared with other bubbles. Heuristically, the chain of transitions $I \rightarrow F \rightarrow A$ can be interpreted as the "dominant" chain in the landscape. The fine-tuned case, $e^{-6}<\eta<e^{6}$, is considered separately, and the result is given by Eq. (54).

I then consider the abundance of Boltzmann brains $(\mathrm{BBs})$ in the FABI landscape (Sec. IIIB). The total number of $\mathrm{BBs}$ is proportional to the total number of Hubble regions ( $H$-regions, or 4 -volumes of order $H^{-4}$ ) in de Sitter bubbles. The coefficient of proportionality is the tiny nucleation rate $\Gamma^{B B}$ of Boltzmann brains, which is of order $\exp \left(-10^{50}\right)$ or smaller. In comparison, ordinary observers occur at a rate of at least 1 per horizon volume. It turns out that (after applying the RV cutoff) the total number of $H$-regions of dS types $F$ or $I$ is approximately equal to the total number of nucleated bubbles of the same type. Hence, the BBs are extremely rare compared with ordinary observers.

In Sec. IV I extend the same calculations to a general landscape with an arbitrary number of vacua. The RV prescription predicts a definite ratio $p(j) / p(k)$ between the number of bubbles of types $j$ and $k$. I derive a formula for the ratio $p(j) / p(k)$ that involves all the parameters of the landscape. With the help of mathematical results derived in Sections IVE and IVF it is possible to show in full generality that the RV measure gives welldefined results that are independent of the initial conditions. Nevertheless, actually performing the required calculations for an arbitrary landscape remains a daunting task. To obtain explicit expressions in a semi-realistic setup, I calculate the bubble abundances for a landscape that contains a single high-energy vacuum and a large number of low-energy vacua. The result is an approximate formula [Eq. (124)] for the ratio $p(j) / p(k)$ expressed directly through the nucleation rates of the landscape.

I also demonstrate in Sec. IV C that the abundance of Boltzmann brains is negligible compared with the abundance of ordinary observers in the same bubble type.

To conclude, the present work demonstrates that the RV measure has attractive features and may be considered a viable candidate for the solution of the measure problem in multiverse cosmology. More work is needed to investigate the dependence of the predictions on the precise details of the definition of the ensemble $E_{n}$. I have developed an extensive mathematical framework for the calculations in the RV prescription and obtained first results for specific landscapes. However, a more powerful approximation scheme is desirable so that the predictions of the RV measure can be more easily obtained for landscapes of general type. Ultimately, the viability of the
RV measure is to be judged by its predictions for cosmological observables in realistic landscapes. These issues will be considered in future publications.

\section{THE RV MEASURE FOR A LANDSCAPE}

The RV measure prescription as formulated in Refs. [50, 51] applies only to the calculation of abundances of terminal bubbles. We will now extend the RV measure to computing arbitrary statistics on a landscape.

We first note that RV measure prescription can be applied, strictly speaking, only to landscapes that contain some terminal bubble types (i.e. vacua from which no further tunneling is possible). However, this limitation is quite benign, for two reasons. First, a landscape without any Minkowski or AdS states is not expected to be realized in any realistic string theory scenario without an exceptional amount of fine-tuning. Second, the previously proposed volume-based and the world-line based measure prescriptions agree for a landscape without terminal bubble types [21, 26]. One may therefore consider the measure problem as solved in such landscapes and turn one's attention to more realistic landscapes where terminal bubble types are present.

Let us take an initial bubble of a nonterminal type $j$ and consider the statistical ensemble $E_{n}(j)$ of all possible evolutions of the initial bubble such that the total number of nucleated bubbles of all types is finite and equals $n$ (not counting the initial bubble). The total number of nucleated bubbles in a multiverse can be finite only if terminal bubbles nucleate everywhere and merge globally to the future of the initial bubble. This can happen by rare chance; however, it is important the total probability of all events in the ensemble $E_{n}(j)$ is always nonzero for any given $n$, so that the ensembles $E_{n}(j)$ are well-defined and nonempty.

The ensemble $E_{n}(j)$ may be described in the language of "transition trees" used in Ref. [21]. The ensemble consists of all trees that have a total number $n+1$ of bubbles, including the initial bubble of type $j$. The trees in $E_{n}(j)$ are finite because all the "outer" leaves are bubbles of terminal types. The motivation for considering the ensemble $E_{n}(j)$ is that a finite but very large tree (with $n$ large) is a controlled approximation to infinite trees that typically occur. Hence, we are motivated to consider $E_{n}(j)$ with $n$ finite but very large.

Note that the ensemble $E_{n}(j)$ differs from the ensemble defined in Ref. [50]; in $E_{n}(j)$ the total number of bubbles of all types is equal to $n$, rather than the total number of terminal bubbles as in Ref. [50]. Thus, the current proposal, which appears more natural, is an extension of that of Ref. [50]. Future work will show whether this technical difference is significant; presently I will investigate the consequences of the current proposal.

Once the ensemble $E_{n}(j)$ is defined, one may consider the statistical distribution of some cosmological observable within the multiverses belonging to the set $E_{n}(j)$. 
For instance, one can count the number of bubbles of some type $k$, or the number of observers within bubbles of type $k$, or the number of observations of some physical process, etc. In a very large multiverse belonging to $E_{n}(j)$ with $n \gg 1$, one may expect that the statistics of observations will be independent of the initial bubble type $j$. Indeed, this will be one of the results of this paper. Hence, let us suppress the argument $j$ and write simply $E_{n}$.

Each multiverse belonging to $E_{n}$ has a naturally defined probability weight, which is simply equal to the probability of realizing that multiverse. This probability weight needs to be taken into account when computing the statistical distributions of observables. The sum of all probability weights of multiverses within $E_{n}$ is equal to the total probability of $E_{n}$, which is exponentially small for large $n$ but always nonzero. Since the multiverses from the ensemble $E_{n}$ are by construction finite, i.e. each multiverse supports only a finite total number of possible observers, we are assured that any statistics we desire to compute on $E_{n}$ will be well-defined.

We can now consider the probability distribution $p\left(Q \mid E_{n}\right)$ of some interesting observable $Q$ within the ensemble $E_{n}$ and take the limit $n \rightarrow \infty$. One expects that the probability distribution $p\left(Q \mid E_{n}\right)$ will have a welldefined limit for large $n$,

$$
p(Q) \equiv \lim _{n \rightarrow \infty} p\left(Q \mid E_{n}\right) .
$$

It was shown in previous work on the RV prescription [50, 51] that the distribution $p(Q)$ is well-defined for a simplest toy landscape as well as in the case of randomwalk eternal inflation. In this work I extend these results to a general landscape scenario. Below (Sec. IVE) I will prove rigorously that the limit (4) indeed exists and is independent of the chosen initial bubble type $j$ as long as the initial bubble is not of terminal type and as long as the landscape is irreducible (every vacuum can be reached from every other non-terminal vacuum by a chain of nucleations). Thus, the distribution $p(Q)$ is unique and well-defined. This distribution is the final result of applying the RV prescription to the observable $Q$.

In practice, it is necessary to compute the distribution $p\left(Q \mid E_{n}\right)$ asymptotically in the limit of large $n$. A direct numerical calculation of probabilities in that limit by enumerating all possible evolution trees is extremely difficult because of the exponential growth of the number of possible evolutions. Instead, I derive explicit formulas for the distribution $p(Q)$ in a generic landscape by evaluating the limit (4) analytically. These formulas are the main result of the present article.

\section{A TOY LANDSCAPE}

I begin by applying the RV measure to a toy landscape with very few vacua. Using this simple example, I develop the computational techniques needed for the practical evaluation of the limit such as Eq. (4). In Sec. IV the same techniques will be extended to a more general landscape with an arbitrary number of vacua.

In Ref. [50] I considered the simplest possible nontrivial landscape: a single dS and two AdS (terminal) vacua. The next least complicated example that can be treated analytically is a toy landscape having two nonterminal and two terminal vacua. This toy landscape was called the "FABI" model in Ref. [24] and consists of the vacua labeled $F, I, A, B$ with the transition diagram $A \leftarrow F \leftrightarrow$ $I \rightarrow B$. In other words, one assumes that the vacuum $F$ ("false" vacuum) can nucleate only bubbles of types $A$ and $I$, the vacuum $I$ ("intermediate" vacuum) can nucleate bubbles of types $F$ and $B$, while $A$ and $B$ are terminal vacua that do not have further nucleations.

To describe the finitely produced probability in this landscape, I use the discrete picture called the "eternal inflation in a box" [50, 53], which is closely related to the "square bubble" approximation [42]. In this picture, one considers the evolution of discrete, causally disjoint homogeneous $H$-regions in discrete time. All possible vacuum types are labeled by $j=1, \ldots, N$. During one time step of order $\delta t=H_{j}^{-1}$, where $H_{j}$ is the local Hubble rate in a given $H$-region of type $j$, the evolution consists of expanding the $H$-region into

$$
e^{3 H_{j} \delta t}=e^{3} \equiv \nu
$$

daughter $H$-regions of type $j$. Each of the daughter $H$ regions has then the probability $\kappa_{j \rightarrow k}$ of changing immediately into an $H$-region of type $k \neq j$; this imitates a nucleation of a horizon-size bubble of type $k$. If no transition has taken place, the daughter $H$-region retains its type $j$. For convenience, we denote by

$$
\kappa_{j \rightarrow j} \equiv 1-\sum_{k \neq j} \kappa_{j \rightarrow k}
$$

the probability of no transitions during one Hubble time. The process of expansion and transition is continued ad infinitum, independently for each resulting $H$-region. The $H$-regions of terminal types will admit no further transitions and will not expand further (except, perhaps, by a fixed amount due to slow-roll inflation occuring immediately after nucleation). An example simulation is shown in Fig. 1

In the remainder of this section I perform explicit calculations of the RV cutoff in the FABI model.

\section{A. Bubble abundances}

The first task is to compute the relative abundance of bubbles of different types. Consider the probability $p\left(n_{\mathrm{tot}}, n_{F}, n_{I}, n_{A}, n_{B} ; k\right)$ of having a finite total number $n_{\text {tot }}$ of bubbles of which $n_{j}$ are of type $j$ (where $j=$ $F, I, A, B)$, if one starts from a single initial $H$-region of type $k(k=F, I)$. By construction, $p \neq 0$ only for $n_{\text {tot }}=n_{F}+n_{I}+n_{A}+n_{B}$. A generating function for this 


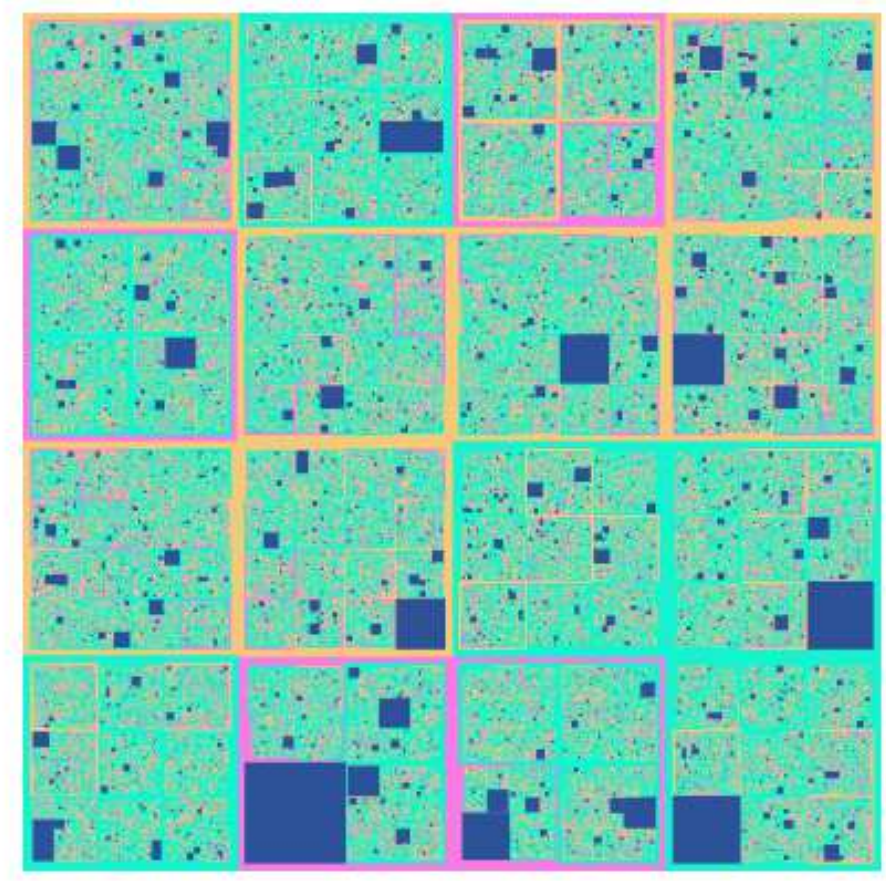

Figure 1: An example simulation of "eternal inflation in a box" in two spatial dimensions. Bubbles ( $H$-regions) are represented in comoving coordinates by squares. Dark shades indicate bubbles of terminal types. Other shades correspond to nested bubbles of various nonterminal ("recyclable") types. For the purposes of visual illustration, nucleation rates were chosen of order one, and colored lines were drawn at bubble boundaries.

probability distribution can be defined by

$$
\begin{aligned}
g\left(z,\left\{q_{j}\right\} ; k\right) & \equiv \sum_{n \geq 0, n_{j} \geq 0} z^{n} p\left(n,\left\{n_{j}\right\} ; k\right) \prod_{j=F, I, A, B} q_{j}^{n_{j}} \\
& \equiv\left\langle z^{n} q_{F}^{n_{F}} q_{I}^{n_{I}} q_{A}^{n_{A}} q_{B}^{n_{B}}\right\rangle_{n<\infty ; k},
\end{aligned}
$$

where the notation $\langle\ldots\rangle_{n<\infty ; k}$ stands for a statistical average restricted to events with a finite total number $n$ of bubbles nucleated to the future of an initial bubble of type $k$.

The generating function $g$ plays a crucial role in the entire calculation. Since we will be only interested in the initial bubbles of types $F$ and $I$, let us denote

$$
F\left(z,\left\{q_{j}\right\}\right) \equiv g\left(z,\left\{q_{j}\right\} ; F\right), \quad I\left(z,\left\{q_{j}\right\}\right) \equiv g\left(z,\left\{q_{j}\right\} ; I\right) .
$$

The generating functions $F$ and $I$ satisfy the following system of nonlinear algebraic equations [50],

$$
\begin{aligned}
F^{\frac{1}{\nu}} & =z q_{A} \kappa_{F A}+z q_{I} \kappa_{F I} I+\kappa_{F F} F, \\
I^{\frac{1}{\nu}} & =z q_{B} \kappa_{I B}+z q_{F} \kappa_{I F} F+\kappa_{I I} I .
\end{aligned}
$$

Here we denoted for brevity $\kappa_{F F} \equiv 1-\kappa_{F A}-\kappa_{F I}$ and $\kappa_{I I} \equiv 1-\kappa_{I B}-\kappa_{I F} ;$ within our assumptions, $\kappa_{F F} \approx 1$ and $\kappa_{I I} \approx 1$. In Eqs. (9)-(10) the generating variable $z$ multiplies only the terms that correspond to changing the type of the $H$-region (which imitates the nucleation of new bubbles) but not the terms $\kappa_{F F} F$ and $\kappa_{I I} I$ that correspond to the eventuality of not changing the type of the $H$-region during one Hubble time.

The nonlinear equations (9)-(10) may have several real-valued solutions, as well as complex-valued solutions that are certainly not of physical interest. In particular, for $z=1$ and $q_{j}=1$ there exists the "trivial" solution $F=I=1$ as well as a nontrivial solution with $F \ll 1$ and $I \ll 1$. Similarly, for $z$ near 0 there exists the solution that approaches $F(0)=I(0)=0$,

$$
F=\left(z q_{A} \kappa_{F A}\right)^{\nu}+O\left(z^{2 \nu-1}\right), I \approx\left(z q_{B} \kappa_{I B}\right)^{\nu}+O\left(z^{2 \nu-1}\right),
$$

as well as the solution

$$
F \approx \kappa_{F F}^{\frac{\nu}{1-\nu}} \approx 1, \quad I \approx \kappa_{I I}^{\frac{\nu}{1-\nu}} \approx 1
$$

and solutions where $F \approx 1$ and $I \approx 0$ and vice versa. It is important to determine the solution branch $F(z), I(z)$ that has the physical significance as the actual generating function of the finitely produced distribution of $\mathrm{H}$ regions.

The functions $F(z), I(z)$ are solutions of algebraic equations and thus are continuous functions of $z$ that are analytic everywhere in complex $z$ plane except for branch cuts. It is easy to see that the solution $F=I=1$ at $z=1, q_{j}=1$ is continuously connected with the solution (12) at $z \approx 0$, while the solution (11) is continued to a solution with $F(z) \ll 1$ and $I(z) \ll 1$ for all $0<z<1$. The values $F(1)$ and $I(1)$ are the probabilities of the events that the evolution of an initial bubble of type $F$ or $I$ ends globally. These probabilities are extremely small and of order $\kappa_{F A}^{\nu}$ and $\kappa_{I B}^{\nu}$ respectively. This is easy to interpret because, for instance, $\kappa_{F A}^{\nu}$ is the probability of nucleating $\nu$ terminal regions at once after one Hubble time within an $H$-region of type $F$. Hence, the generating functions for the finitely produced distribution of bubbles are given by the solution branch having $F \ll 1$ and $I \ll 1$ rather than by the solution $F=I=1$ at $z=1$. More precisely, the physically meaningful solution $F(z), I(z)$ is selected by the asymptotic behavior (11). This argument removes the ambiguity inherent in solving Eqs. (91)-(10). Below we refer to the branch of solutions $F(z), I(z)$ connected to the nontrivial solution (11) near $z=0$ as the "main branch."

Once the main branch of the generating functions $g\left(z,\left\{q_{j}\right\} ; k\right)$ are known, one can express the mean number of bubbles of type $i(i=F, I, A, B)$ at a fixed total number $n_{\text {tot }}$ as

$$
p\left(i \mid n_{\mathrm{tot}}\right) \equiv \frac{\left\langle n_{i}\right\rangle_{n_{\mathrm{tot}}}}{n_{\mathrm{tot}}}=\frac{\partial_{z}^{n_{\mathrm{tot}}} \partial_{q_{i}} g\left(z=0,\left\{q_{j}=1\right\} ; k\right)}{n_{\mathrm{tot}} \partial_{z}^{n_{\mathrm{tot}}} g\left(z=0,\left\{q_{j}=1\right\} ; k\right)} .
$$

One expects that the limit of this ratio at $n_{\text {tot }} \rightarrow \infty$ will be independent of the initial bubble type $k$ since the ensemble $E_{n_{\text {tot }}}$ will consist of $H$-regions having a very long evolution, so that the initial conditions are forgotten. Below I will show explicitly that this is indeed the case. 
It is more convenient to compute the ratios of the number of bubbles of types $i$ and $i^{\prime}$ at fixed $n_{\text {tot }}$,

$$
\frac{p\left(i \mid n_{\mathrm{tot}}\right)}{p\left(i^{\prime} \mid n_{\mathrm{tot}}\right)}=\left.\frac{\partial_{z}^{n_{\mathrm{tot}}} \partial_{q_{i}} g}{\partial_{z}^{n_{\mathrm{tot}}} \partial_{q_{i^{\prime}}} g}\right|_{z=0,\left\{q_{j}=1\right\}} .
$$

Then one only needs to compute derivatives $\partial_{q_{i}} g \equiv g_{, q_{i}}$ evaluated at $q_{j}=1$. These derivatives satisfy a system of linear equations that can be easily derived from Eqs. (9)(10). For instance, the derivatives $F_{, q_{A}}$ and $I_{, q_{A}}$ satisfy

$$
\begin{aligned}
& \frac{1}{\nu} F^{\frac{1}{\nu}-1} F_{, q_{A}}=z \kappa_{F A}+z \kappa_{F I} I_{, q_{A}}+\kappa_{F F} F_{, q_{A}}, \\
& \frac{1}{\nu} I^{\frac{1}{\nu}-1} I_{, q_{A}}=z \kappa_{I F} F_{q_{A}}+\kappa_{I I} I_{q_{A}} .
\end{aligned}
$$

Rewriting these equations in a matrix form, we obtain

$$
\left(\begin{array}{cc}
\frac{1}{\nu} F^{\frac{1}{\nu}-1}-\kappa_{F F} & -z \kappa_{F I} \\
-z \kappa_{I F} & \frac{1}{\nu} I^{\frac{1}{\nu}-1}-\kappa_{I I}
\end{array}\right)\left[\begin{array}{c}
F_{, q_{A}} \\
I_{, q_{A}}
\end{array}\right]=\left[\begin{array}{c}
z \kappa_{F A} \\
0
\end{array}\right] .
$$

The coefficients of the $z$-dependent matrix

$$
\hat{M}(z) \equiv\left(\begin{array}{cc}
\frac{1}{\nu} F^{\frac{1}{\nu}-1}-\kappa_{F F} & -z \kappa_{F I} \\
-z \kappa_{I F} & \frac{1}{\nu} I^{\frac{1}{\nu}-1}-\kappa_{I I}
\end{array}\right)
$$

are the main branch of solutions of the nonlinear equations (91)-(10) at $q_{j}=1$ but at arbitrary $z$.

One can verify using Eq. (11) that the matrix $\hat{M}(z)$ is invertible near $z=0$. Hence the solution of Eq. (17) can be written, at least within some range of $z$ where $\hat{M}(z)$ remains invertible, as

$$
\left[\begin{array}{c}
F_{, q_{A}} \\
I_{, q_{A}}
\end{array}\right]=\hat{M}^{-1}(z)\left[\begin{array}{c}
z \kappa_{F A} \\
0
\end{array}\right] .
$$

Similarly, the derivatives $F_{q_{F}}$ and $I_{q_{F}}$ satisfy the equations

$$
\hat{M}(z)\left[\begin{array}{c}
F_{, q_{F}} \\
I_{, q_{F}}
\end{array}\right]=\left[\begin{array}{c}
0 \\
z \kappa_{I F} F
\end{array}\right],
$$

whose solution is

$$
\left[\begin{array}{c}
F_{, q_{F}} \\
I_{, q_{F}}
\end{array}\right]=\hat{M}^{-1}(z)\left[\begin{array}{c}
0 \\
z \kappa_{I F} F
\end{array}\right] .
$$

Other generating functions can be expressed in the same manner.

One could in principle obtain a numerical solution for $F_{, q_{A}}(z), I_{, q_{A}}(z)$, and all the other generating functions at any given value of $z$. However, the numerical solution is not particularly useful at this point because the next step in the calculation is the evaluation of Eq. (14) in the limit of very large $n_{\text {tot }}$, for instance,

$$
\frac{p\left(A \mid n_{\mathrm{tot}}\right)}{p\left(F \mid n_{\mathrm{tot}}\right)}=\left.\frac{\partial_{z}^{n_{\text {tot }}} F_{, q_{A}}}{\partial_{z}^{n_{\text {tot }}} F_{, q_{F}}}\right|_{z=0} .
$$

It is generally not feasible to compute the $n$-th derivative of a numerically obtained function in the limit $n \rightarrow \infty$, because the unavoidable round-off errors are amplified by a fixed factor with each successive numerical differentiation. Therefore, we need a way to evaluate the ratio (14) in the limit $n_{\text {tot }} \rightarrow \infty$ without using numerics. Indeed we will be able to compute the ratio $p(A) / p(F)$. We will also show that, for instance,

$$
\frac{p(A)}{p(F)}=\left.\lim _{n \rightarrow \infty} \frac{\partial_{z}^{n} F_{, q_{A}}}{\partial_{z}^{n} F_{, q_{F}}}\right|_{z=0}=\left.\lim _{n \rightarrow \infty} \frac{\partial_{z}^{n} I_{, q_{A}}}{\partial_{z}^{n} I_{, q_{F}}}\right|_{z=0} ;
$$

in other words, that the final RV-regulated ratio $p(A) / p(F)$ is independent of whether the initial bubble is of type $F$ or of type $I$.

To proceed, we use the fact that $F_{, q_{A}}(z), I_{, q_{A}}(z)$, $F_{, q_{F}}(z)$, etc. are analytic functions of the parameter $z$. The asymptotic growth of high-order derivatives of an analytic function $f(z)$ is determined by the location of the singularities of $f(z)$ in the complex $z$ plane. The required result can be derived by the following elementary argument: Consider the derivative $d^{n} f / d z^{n}$ at $z=0$, and assume that $f(z)$ admits an expansion around the singularity $z_{*}$ nearest to $z=0$, such as

$$
f(z)=c_{0}+c_{1}\left(z-z_{*}\right)^{s}+\ldots
$$

where $s \neq 0,1,2, \ldots$ is the power of the leading-order singularity, and the omitted terms are either higher powers of $z-z_{*}$ or singularities at points $z_{*}^{\prime}$ located further away from $z=0$. The singularity structure (24) yields the large- $n$ asymptotics

$$
\left.\frac{d^{n} f}{d z^{n}}\right|_{z=0} \approx c_{1}\left(-z_{*}\right)^{s} \frac{\Gamma(n-s)}{\Gamma(-s)} z_{*}^{-n}+\ldots
$$

It can be seen from this formula that any other singular point $z_{*}^{\prime}$ located further away from $z=0$ gives a contribution that is smaller by the factor $\left|z_{*}^{\prime} / z_{*}\right|^{s-n}$. The contribution of a subdominant singularity of the form $\left(z-z_{*}\right)^{s^{\prime}}$, i.e. at the same point $z=z_{*}$ but with a higher power $s^{\prime}>s$, is suppressed, in comparison with the term in Eq. (25), by the factor

$$
\frac{\Gamma\left(n-s^{\prime}\right)}{\Gamma(n-s)} \frac{\Gamma(-s)}{\Gamma\left(-s^{\prime}\right)} \approx \frac{1}{(n-1)^{s^{\prime}-s}} \frac{\Gamma(-s)}{\Gamma\left(-s^{\prime}\right)} .
$$

It is clear that the terms omitted from Eq. (25) indeed give subleading contributions at large $n$, and so Eq. (25) is indeed the leading asymptotic term at $n \rightarrow \infty$.

We now need to determine the location of the singularities of $F_{, q_{A}}(z), I_{, q_{A}}(z)$, etc., as functions of $z$. This task is much simplified once we observe that all these quantities are expressed through the inverse matrix $\hat{M}^{-1}(z)$, and hence it remains to analyze the singularities of $\hat{M}^{-1}(z)$. That matrix can be singular at some value $z=z_{*}$ either because some of the coefficients of $\hat{M}(z)$ are singular, or because the matrix $\hat{M}(z)$ is degenerate (noninvertible) at $z=z_{*}$. The coefficients of $\hat{M}(z)$ depend on solutions $F(z), I(z)$ of algebraic equations (9)-(10) at $q_{j}=1$ and 
thus cannot be divergent as functions of $z$. These coefficients can be singular only in that some derivative in $z$ diverges. The derivatives $F_{, z}$ and $I_{, z}$ satisfy the equations

$$
\hat{M}(z)\left[\begin{array}{c}
F_{, z} \\
I_{, z}
\end{array}\right]=\left[\begin{array}{c}
\kappa_{F A}+\kappa_{F I} I \\
\kappa_{I B}+\kappa_{I F} F
\end{array}\right] .
$$

Therefore, $F_{, z}$ and $I_{, z}$ diverge only for those $z$ for which the matrix $\hat{M}(z)$ is degenerate. For these $z$, all the derivatives $F_{, z}, I_{, z}, F_{q_{A}}$, etc. will be divergent at the same time since they are all proportional to the inverse matrix $\hat{M}^{-1}(z)$.

We note that the matrix $\hat{M}(z)$ is the Jacobian of the nonlinear system (9)-(10). As long as $\hat{M}(z)$ is nondegenerate, all the different branches of the solutions $F(z), I(z)$ do not meet and remain smooth functions of $z$. As we noted above, the "main" branch $F(z), I(z)$ is the one connected to the nontrivial solution (111) at $z \approx 0$. The matrix $\hat{M}(z)$ is nondegenerate near $z=0$; therefore, the solutions $F(z), I(z)$ remain smooth functions of $z$ for all $z$ such that $\hat{M}(z)$ is nondenegerate. We conclude that the only possible singularities of $F(z), I(z)$ are those values $z=z_{*}$ where $\operatorname{det} \hat{M}(z)=0$.

Below it will be shown that the behavior of $\operatorname{det} \hat{M}(z)$ near $z=z_{*}$ is

$$
\operatorname{det} \hat{M}(z) \approx c_{1} \sqrt{z_{*}-z}
$$

i.e. of the form (24) with $c_{0}=0$ and $s=\frac{1}{2}$. It now follows from Eqs. (19) and (21) that the derivatives such as $F_{, q_{A}}(z), I_{, q_{A}}(z), F_{q_{F}}(z)$, etc., all diverge at $z=z_{*}$ with the same asymptotic behavior, namely proportional to $\left(z_{*}-z\right)^{-1 / 2}$. We may express the inverse matrix as

$$
\begin{aligned}
\hat{M}^{-1}(z) & =\frac{1}{\operatorname{det} \hat{M}(z)} \hat{\tilde{M}}(z), \\
\hat{\tilde{M}}(z) & \equiv\left(\begin{array}{cc}
\frac{1}{\nu} I^{\frac{1}{\nu}-1}-\kappa_{I I} & z \kappa_{F I} \\
z \kappa_{I F} & \frac{1}{\nu} F^{\frac{1}{\nu}-1}-\kappa_{F F}
\end{array}\right),
\end{aligned}
$$

where we introduced the algebraic cofactor matrix $\hat{\tilde{M}} \equiv$ $\hat{M}^{-1} \operatorname{det} \hat{M}$, which is nonsingular at $z=z_{*}$. It then follows from Eq. (19) that the asymptotic behavior of the functions $F_{, q_{A}}(z)$ and $I_{, q_{A}}(z)$ near $z=z_{*}$ is given by

$$
\begin{aligned}
{\left[\begin{array}{c}
F_{, q_{A}} \\
I_{, q_{A}}
\end{array}\right] } & \approx \frac{1}{c_{1} \sqrt{z_{*}-z}} \hat{\tilde{M}}\left(z_{*}\right)\left[\begin{array}{c}
z_{*} \kappa_{F A} \\
0
\end{array}\right] \\
& =\frac{1}{c_{1} \sqrt{z_{*}-z}}\left[\begin{array}{c}
\left(\frac{1}{\nu} I^{\frac{1}{\nu}-1}-\kappa_{I I}\right) z_{*} \kappa_{F A} \\
z_{*}^{2} \kappa_{I F} \kappa_{F A}
\end{array}\right] .
\end{aligned}
$$

Similarly, using Eq. 21) we find near $z=z_{*}$

$$
\begin{aligned}
{\left[\begin{array}{c}
F_{, q_{F}} \\
I_{, q_{F}}
\end{array}\right] } & \approx \frac{1}{c_{1} \sqrt{z_{*}-z}} \hat{\tilde{M}}\left(z_{*}\right)\left[\begin{array}{c}
0 \\
z_{*} \kappa_{I F} F
\end{array}\right] \\
& =\frac{1}{c_{1} \sqrt{z_{*}-z}}\left[\begin{array}{c}
z_{*}^{2} \kappa_{F I} \kappa_{I F} F \\
\left(\frac{1}{\nu} F^{\frac{1}{\nu}-1}-\kappa_{F F}\right) z_{*} \kappa_{I F} F
\end{array}\right] .
\end{aligned}
$$

Using Eq. (25) and noticing that all $n$-dependent factors are the same in $\partial_{z}^{n} F_{, q_{A}}$ and other such derivatives, we conclude that

$$
\begin{aligned}
& \left.\lim _{n \rightarrow \infty} \frac{\partial_{z}^{n} F_{q_{A}}}{\partial_{z}^{n} F_{q_{F}}}\right|_{z=0}=F^{\frac{1}{\nu}} \frac{\left(\frac{1}{\nu} I_{*}^{\frac{1}{\nu}-1}-\kappa_{I I}\right) \kappa_{F A}}{z_{*} \kappa_{F I} \kappa_{I F} F_{*}} \\
& \left.\lim _{n \rightarrow \infty} \frac{\partial_{z}^{n} I_{q_{A}}}{\partial_{z}^{n} I_{q_{F}}}\right|_{z=0}=\frac{z_{*} \kappa_{F A}}{\left(\frac{1}{\nu} F_{*}^{\frac{1}{\nu}-1}-\kappa_{F F}\right) F_{*}}
\end{aligned}
$$

It is important that the two limits above are equal; this is so because the condition $\operatorname{det} \hat{M}\left(z_{*}\right)=0$ yields

$$
\operatorname{det} \hat{M}=\left[\frac{F_{*}^{\frac{1}{\nu}-1}}{\nu}-\kappa_{F F}\right]\left[\frac{I_{*}^{\frac{1}{\nu}-1}}{\nu}-\kappa_{I I}\right]-z_{*}^{2} \kappa_{F I} \kappa_{I F}=0,
$$

where we denoted $F_{*} \equiv F\left(z_{*}\right), I_{*} \equiv I\left(z_{*}\right)$ for brevity. It follows that the ratio of $A$-bubbles to $F$-bubbles is independent of whether the initial bubble is of type $F$ or of type $I$. In other words, the RV-regulated ratio of $A$-bubbles to $F$-bubbles is

$$
\frac{p(A)}{p(F)}=\frac{z_{*} \kappa_{F A}}{\left(\frac{1}{\nu} F_{*}^{\frac{1}{\nu}-1}-\kappa_{F F}\right) F_{*}}
$$

independently of the initial bubble type. Below (Sec.IVE) the independence of initial conditions will be rigorously proved for a general landscape using mathematical techniques of the theory of nonnegative matrices. Presently we have shown this independence using explicit formulas available for the toy landscape under consideration.

In a similar way, we find the RV-regulated ratio of $A$ bubbles to $B$-bubbles,

$$
\frac{p(A)}{p(B)}=\frac{z_{*} \kappa_{F A} \kappa_{I F}}{\left(\frac{1}{\nu} F_{*}^{\frac{1}{\nu}-1}-\kappa_{F F}\right) \kappa_{I B}}
$$

and the ratio of $F$-bubbles to $I$-bubbles,

$$
\frac{p(F)}{p(I)}=\frac{\left(\frac{1}{\nu} F_{*}^{\frac{1}{\nu}-1}-\kappa_{F F}\right) F_{*}}{z_{*} \kappa_{F I} I_{*}} .
$$

It remains to compute $z_{*}, F_{*}, I_{*}$ and to justify Eq. (28). We will do this using the explicit form of the matrix $\hat{M}(z)$. To determine $z_{*}, F_{*}$, and $I_{*}$, we need to solve Eq. (35) simultaneously with the equations

$$
\begin{gathered}
F_{*}^{\frac{1}{\nu}}=z_{*} \kappa_{F A}+z_{*} \kappa_{F I} I_{*}+\kappa_{F F} F_{*}, \\
I_{*}^{\frac{1}{\nu}}=z_{*} \kappa_{I B}+z_{*} \kappa_{I F} F_{*}+\kappa_{I I} I_{*},
\end{gathered}
$$

the latter two being Eqs. (9)-(10) after setting $q_{j}=1$. We are interested in the solution $z_{*}$ closest to $z=0$. By definition (7), the generating functions are nonsingular for $|z| \leq 1$; hence, the only possible values of $z_{*}$ are in the domain $|z|>1$ in the complex plane. 
Moreover, we can show that the solutions $F(z), I(z)$ are growing functions of $z$ for real $z<z_{*}$. Initially at $z \approx 0$ these functions have the form (11) and hence are positive. The determinant $\operatorname{det} \hat{M}$ shown in Eq. (35) is also positive for $z<z_{*}$. One can then find from Eq. (30) that the algebraic cofactor matrix $\hat{\tilde{M}}$ and hence the inverse matrix $\hat{M}^{-1}$ has all positive elements for those $z$. It is then evident from Eq. (27) that $\partial_{z} F>0$ and $\partial_{z} I>0$ as long as the values of $F$ and $I$ are themselves positive. Therefore, the solutions $F(z), I(z)$ are positive and growing functions of $z$ as long as $\operatorname{det} \hat{M}(z)>0$.

We may thus visualize the behavior of $\operatorname{det} \hat{M}(z)$ as $z$ grows. Both $F(z)$ and $I(z)$ will grow with $z$, so that the terms in square brackets diminish while the second term, $z_{*}^{2} \kappa_{F I} \kappa_{I F}$, grows. Eventually the product of the square brackets in Eq. (35) will be balanced by the second term, and the determinant will vanish. We need to determine the smallest value $z=z_{*}$ for which $\operatorname{det} \hat{M}(z)=0$.

Since the physically significant branch of the solution involves always very small values $F(z)$ and $I(z)$ for $|z| \leq$ 1 , while other (unphysical) branches have either $F$ or $I$ approximately equal to 1 , it is reasonable to assume that $F\left(z_{*}\right) \ll 1$ and $I\left(z_{*}\right) \ll 1$ also at $z=z_{*}$ (the selfconsistency of this assumption will be confirmed by later calculations). Then the terms $\kappa_{F F} F_{*} \approx F_{*}$ and $\kappa_{I I} I_{*} \approx$ $I_{*}$ can be disregarded in comparison with $F_{*}^{1 / \nu}$ and $I_{*}^{1 / \nu}$ in Eqs. (39)-(40). In this approximation, we can simplify Eqs. (39)- 40 to

$$
\begin{gathered}
F_{*}^{\frac{1}{\nu}}=z_{*} \kappa_{F A}+z_{*} \kappa_{F I} I_{*}, \\
I_{*}^{\frac{1}{\nu}}=z_{*} \kappa_{I B}+z_{*} \kappa_{I F} F_{*},
\end{gathered}
$$

while Eq. (35) becomes

$$
\left(\kappa_{F A}+\kappa_{F I} I_{*}\right)\left(\kappa_{I B}+\kappa_{I F} F_{*}\right)=\nu^{2} \kappa_{F I} \kappa_{I F} F_{*} I_{*} .
$$

The ratios (36)-(38) are also simplified and can be written more concisely as

$$
\begin{aligned}
p(A): p(I) & : p(F): p(B)=\kappa_{F A}:\left(\kappa_{F I} I_{*}\right) \\
& : \frac{\kappa_{F A}+\kappa_{F I} I_{*}}{\nu}: \kappa_{I B} \frac{\kappa_{F A}+\kappa_{F I} I_{*}}{\nu \kappa_{I F} F_{*}} .
\end{aligned}
$$

To determine $z_{*}$, we will obtain an explicit approximation for the main branch $F(z), I(z)$ for all $z$. For small enough $z$, the solutions of Eqs. (39)-(40) are well approximated by Eq. (11),

$$
F(z) \approx\left(z \kappa_{F A}\right)^{\nu}, \quad I(z) \approx\left(z \kappa_{I B}\right)^{\nu}
$$

These solutions are obtained under the assumption that the terms $z \kappa_{F A}$ and $z \kappa_{I B}$ are numerically small $\left(z \kappa_{F A} \ll\right.$ $1, z \kappa_{I B} \ll 1$ ) and yet dominant in Eqs. (39)-(40). These terms only remain dominant as long as

$$
\kappa_{F A} \gg \kappa_{F I} I(z), \quad \kappa_{I B} \gg \kappa_{I F} F(z) .
$$

Substituting Eq. (11) for $F(z)$ and $I(z)$ into Eq. (46), we obtain the conditions

$$
z \ll \kappa_{I B}^{-1}\left(\frac{\kappa_{F A}}{\kappa_{F I}}\right)^{\frac{1}{\nu}}, \quad z \ll \kappa_{F A}^{-1}\left(\frac{\kappa_{I B}}{\kappa_{I F}}\right)^{\frac{1}{\nu}} .
$$

We need to check whether $\operatorname{det} \hat{M}(z)$ could vanish already for some $z_{*}$ within the range (47). Using Eq. (43) in the regime (46), we find

$$
z_{*} \approx\left[\left(\kappa_{F A} \kappa_{I B}\right)^{\nu-1} \nu^{2} \kappa_{F I} \kappa_{I F}\right]^{-\frac{1}{2 \nu}} .
$$

This value is within the range (47) only if the following simultaneous inequalities hold,

$$
e^{-6} \equiv \frac{1}{\nu^{2}} \ll\left(\frac{\kappa_{I B}}{\kappa_{F A}}\right)^{\nu+1} \frac{\kappa_{F I}}{\kappa_{I F}} \ll \nu^{2} \equiv e^{6} .
$$

Let us denote by $\eta$ the quantity in Eq. (49),

$$
\eta \equiv\left(\frac{\kappa_{I B}}{\kappa_{F A}}\right)^{\nu+1} \frac{\kappa_{F I}}{\kappa_{I F}}
$$

then Eq. (49) becomes simply $|\ln \eta|<6$. One would expect that $\eta$ is generically either very large or very small, so the inequalities (49) can hold only in a fine-tuned landscape. Additionally, we need to require

$$
z_{*} \ll \min \left(\frac{1}{\kappa_{F A}}, \frac{1}{\kappa_{I B}}\right),
$$

which entails

$$
\frac{\kappa_{I B}}{\kappa_{I F}} \ll \nu \sqrt{\eta}, \quad \frac{\kappa_{F A}}{\kappa_{F I}} \ll \frac{\nu}{\sqrt{\eta}} .
$$

However, this requirement is weaker than Eq. (49) since typically $\kappa_{F A} \ll \kappa_{F I}$ and $\kappa_{I B} \ll \kappa_{I F}$, so

$$
z_{*} \ll \kappa_{I B}^{-1}\left(\frac{\kappa_{F A}}{\kappa_{F I}}\right)^{\frac{1}{\nu}} \ll \kappa_{I B}^{-1} ; z \ll \kappa_{F A}^{-1}\left(\frac{\kappa_{I B}}{\kappa_{I F}}\right)^{\frac{1}{\nu}} \ll \kappa_{F A}^{-1} .
$$

Let us assume, for the moment, that Eqs. (49) and (52) hold. Then we use Eq. (44) to obtain

$$
p(A): p(I): p(F): p(B) \approx 1: \frac{\sqrt{\eta}}{\nu}: \frac{1}{\nu}: \sqrt{\eta} .
$$

Due to the fine-tuning assumption (49), these ratios are all within the interval $\left[\nu^{-2}, \nu^{2}\right]=\left[e^{-6}, e^{6}\right]$.

Having considered the fine-tuned case, let us now turn to the generic case. Generically one would expect that the quantity $\eta$ is either extremely large or extremely small, and in any case outside the logarithmically narrow range $\left[e^{-6}, e^{6}\right]$. In that case, one of the inequalities (49) does not hold; generically, either $\eta \ll \nu^{-2}$ or $\eta \gg \nu^{2}$. It follows that $\operatorname{det} \hat{M}(z) \neq 0$ for all $z$ within the range (47). To reach the value $z_{*}$ at which $\operatorname{det} \hat{M}\left(z_{*}\right)=0$, we need to increase $z$ further, until one of the terms $z \kappa_{F I} I$ or $z \kappa_{I F} F$ 
in Eqs. (39) - (40) becomes dominant and the solution (45) becomes invalid.

It is impossible that both the terms $z \kappa_{F I} I$ and $z \kappa_{I F} F$ are dominant in Eqs. (39)-(40) at $z=z_{*}$ because then Eq. (43) would yield a contradiction,

$$
\kappa_{F I} I_{*} \kappa_{I F} F_{*}=\nu^{2} \kappa_{F I} \kappa_{I F} F_{*} I_{*} .
$$

Hence, the determinant $\operatorname{det} \hat{M}(z)$ first vanishes at the value $z=z_{*}$ such that only one of those terms is dominant in its respective equation. Without loss of generality, we may relabel the vacua $(F \leftrightarrow I, A \leftrightarrow B)$ such that the term $z \kappa_{I F} F$ becomes dominant in Eq. (40) while the term $z \kappa_{F A}$ is still dominant in Eq. (39). This is equivalent to assuming $\eta \ll \nu^{-2}$. In the range of $z$ for which this is the case,

$$
z \kappa_{F I} I(z) \ll z \kappa_{F A} \ll 1 ; \quad z \kappa_{I B} \ll z \kappa_{I F} F(z) \ll 1,
$$

the approximate solution of Eqs. (39)-(40) can be written as

$$
F(z) \approx\left(z \kappa_{F A}\right)^{\nu} ; I(z) \approx\left(z \kappa_{I F} F\right)^{\nu}=\kappa_{F A}^{\nu^{2}} \kappa_{I F}^{\nu} z^{(\nu+1) \nu} .
$$

With these values of $F(z)$ and $I(z)$, the consistency requirement (56) yields the following range of $z$,

$$
\frac{\kappa_{I B}}{\kappa_{I F} \kappa_{F A}^{\nu}} \ll z^{\nu} \ll\left[\kappa_{F A}^{\nu^{2}-1} \kappa_{F I} \kappa_{I F}^{\nu}\right]^{-\frac{1}{\nu+1}} .
$$

This range is nonempty if

$$
\eta \equiv\left(\frac{\kappa_{I B}}{\kappa_{F A}}\right)^{\nu+1} \frac{\kappa_{F I}}{\kappa_{I F}} \ll 1,
$$

which is indeed one of the two possible ways that the fine-tuning (49) can fail. Having assumed that the above condition holds, we need to determine the value of $z_{*}$ and check that it belongs to the range (56). Using Eq. (43) in the regime (56), we obtain

$$
z_{*} \approx\left[\nu^{2} \kappa_{I F}^{\nu} \kappa_{F I} \kappa_{F A}^{\nu^{2}-1}\right]^{-\frac{1}{\nu(\nu+1)}} .
$$

Substituting this value into the inequalities (58), we find the condition

$$
\eta \ll \frac{1}{\nu^{2}} \ll 1,
$$

which holds identically under the current assumption, $\eta \ll \nu^{-2}$. (The relabeling $F \leftrightarrow I, A \leftrightarrow B$ is necessary if the opposite case, $\eta \gg \nu^{2}$, holds.) Therefore, $z_{*}$ is within the regime (56), and our approximations are self-consistent, yielding

$$
F_{*} \approx\left(z_{*} \kappa_{F A}\right)^{\nu}=\frac{\kappa_{F A}}{\left[\nu^{2} \kappa_{I F}^{\nu} \kappa_{F I}\right]^{\frac{1}{\nu+1}}}, \quad I_{*} \approx \frac{\kappa_{F A}}{\nu^{2} \kappa_{F I}} .
$$

The ratios (44) become

$$
p(A): p(I): p(F): p(B) \approx 1: \frac{1}{\nu^{2}}: \frac{1}{\nu}:\left[\frac{\eta}{\nu^{\nu-1}}\right]^{\frac{1}{\nu+1}} .
$$

This is the result of applying the RV prescription to a generic $F A B I$ toy landscape in the second regime.

It remains to justify the statement of Eq. (28). Below in Sec. IVE I will demonstrate that the property (28) holds for a general landscape. Here only a simple argument is presented to illustrate this property for Eqs. (41)(42). Using those equations, we can express $I_{*}$ through $F_{*}$ and derive a closed algebraic equation for $F_{*}$,

$$
F_{*}=z^{\nu}\left[\kappa_{F A}+\kappa_{F I} z^{\nu}\left(\kappa_{I B}+\kappa_{I F} F_{*}\right)^{\nu}\right]^{\nu} \equiv f\left(z ; F_{*}\right) .
$$

The solution $F(z)$ is given by the intersection of the line $y=F$ and the curve $y=f(z ; F)$ in the $y-F$ plane. The function $f(z ; F)$ is convex in $F$ for $F>0, z>0$; hence, there will be a value $z=z_{*}$ for which the curve $f\left(z_{*} ; F\right)$ is tangent to the line $y=F$, i.e. $f_{, F}\left(z_{*} ; F_{*}\right)=1$. This value of $z_{*}$ will then implicitly determine $F_{*}$. For values $F \approx F_{*}, z \approx z_{*}$ the dependence of $F(z)$ on $z$ will exhibit the singularity behavior of the type $\sqrt{z_{*}-z}$. To see this formally, we may expand

$$
\begin{aligned}
F & =f(z ; F) \approx F_{*}+f_{, F}\left(F-F_{*}\right)+\frac{1}{2} f_{, F F}\left(F-F_{*}\right)^{2} \\
& +f_{, z}\left(z-z_{*}\right) .
\end{aligned}
$$

Since $f_{, F}\left(z_{*} ; F_{*}\right)=1$, we obtain

$$
F \approx F_{*}+\left.\frac{2 f_{, z}}{f_{, F F}}\right|_{F_{*}, z_{*}} \sqrt{z_{*}-z} .
$$

This shows explicitly the singularity structure of the form (24). The determinant of the matrix $\hat{M}$ is a smooth function of $F(z)$ and $I(z)$ near $z=z_{*}$. Expressing $\operatorname{det} \hat{M}$ as a function only of $F$, we obtain for $z \approx z_{*}$ the required formula (28),

$$
\left.\operatorname{det} \hat{M}(z) \approx\left(F-F_{*}\right) \frac{d}{d F}\right|_{F_{*}} \operatorname{det} \hat{M} \propto \sqrt{z_{*}-z} .
$$

\section{B. "Boltzmann brains"}

Let us now use the same techniques to compute the relative abundance of "Boltzmann brain" observers to ordinary observers.

We need to introduce an appropriate set of generating functions. "Boltzmann brains" can be created with a fixed probability per unit 4-volume, unlike ordinary observers who can appear only within a narrow interval of time after creation of a given bubble. Let us therefore compare the total number of bubbles $n_{j}$ with the total number of 4 -volumes $H_{j}^{-4}$ in bubbles of type $j$.

To be specific, let us fix $j=F$ (no Boltzmann brains can be expected in a terminal vacuum). Consider the probability $p\left(n_{\mathrm{tot}}, n_{F}, N_{F} ; k\right)$ of having a finite total number $n_{\text {tot }}$ of bubbles of which $n_{F}$ are of type $F$ and $N_{F}$ is the total number of $H$-regions of type $F$, if one starts from a single initial $H$-region of type $k(k=F, I)$. 
The number of "Boltzmann brains" in bubbles of type $F$ is proportional to $N_{F}$ with a small proportionality constant, $\kappa_{F}^{B B}$, which we will include at the end of the calculation. A generating function $g$ for the probability distribution $p$ can be defined by

$$
\begin{aligned}
g(z, q, r ; k) & \equiv \sum_{n, n_{F}, N_{F} \geq 0} z^{n} q^{n_{F}} r^{N_{F}} p\left(n, n_{F}, N_{F} ; k\right) \\
& \equiv\left\langle z^{n} q^{n_{F}} r^{N_{F}}\right\rangle_{n<\infty ; k} .
\end{aligned}
$$

For the initial bubble types $k=F$ and $k=I$, let us denote for brevity

$$
F(z, q, r) \equiv g(z, q, r ; F), \quad I(z, q, r) \equiv g(z, q, r ; I) .
$$

The generating functions $F$ and $I$ satisfy the following system of equations,

$$
\begin{aligned}
F^{\frac{1}{\nu}} & =z \kappa_{F A}+z \kappa_{F I} I+r \kappa_{F F} F, \\
I^{\frac{1}{\nu}} & =z \kappa_{I B}+z r q \kappa_{I F} F+\kappa_{I I} I .
\end{aligned}
$$

These equations differ from the analogous Eqs. (91)-(10) in that the generating parameter $q$ appears when a new bubble of type $F$ is created, while the parameter $r$ appears every time a new $H$-region of type $F$ is created, which can happen via Hubble expansion of old $F$-bubbles as well as through nucleation of new $F$-bubbles.

We would like to compare the mean number of $\mathrm{H}$ regions of type $F$ with the mean number of new bubbles of type $F$, so we compute (e.g. starting with $I$-bubbles)

$$
\frac{\left\langle N_{F}\right\rangle_{n ; I}}{\left\langle n_{F}\right\rangle_{n ; I}}=\left.\frac{\partial_{z}^{n} \partial_{r} I}{\partial_{z}^{n} \partial_{q} I}\right|_{z=0, r=1, q=1}
$$

and take the limit $n \rightarrow \infty$. Taking the limit as $n \rightarrow \infty$ of the ratio $\partial_{z}^{n} F_{, r} / \partial_{z}^{n} F_{, q}$ will yield the same result, but the limit of Eq. (72) is more straightforwardly analyzed.

As before, we first obtain the equations for the derivatives $F_{, q}, I_{, q}, F_{, r}, I_{, r}$ at $q=r=1$ from Eqs. (70)-(71). The derivatives $F_{, q}$ and $I_{, q}$ satisfy the same equations as before, namely Eq. (20), while $F_{, r}$ and $I_{, r}$ satisfy

$$
\hat{M}(z)\left[\begin{array}{c}
F_{, r} \\
I_{, r}
\end{array}\right]=\left[\begin{array}{c}
\kappa_{F F} F \\
z \kappa_{I F} F
\end{array}\right],
$$

whose solution is

$$
\left[\begin{array}{c}
F_{, r} \\
I_{, r}
\end{array}\right]=\hat{M}^{-1}(z)\left[\begin{array}{c}
\kappa_{F F} F \\
z \kappa_{I F} F
\end{array}\right] .
$$

Using the same arguments as in the previous section, we evaluate the limit of Eq. (72),

$$
\left.\lim _{n \rightarrow \infty} \frac{\partial_{z}^{n} \partial_{r} I}{\partial_{z}^{n} \partial_{q} I}\right|_{z=0, r=1, q=1}=\nu F_{*}^{1-\frac{1}{\nu}} \kappa_{F F}+1 .
$$

To analyze this simple result, we do not actually need to use the complicated decision procedure of Sec. III. Since
$\kappa_{F F} \approx 1$ and $F_{*}<1$ in any case, ${ }^{3}$ the value (75) is bounded from above by $\nu+1$, which is not a large number. Hence, the mean total number of $H$-regions of type $F$ is at most $\nu+1$ times larger than the mean total number of bubbles of type $F$. (Both numbers are finite in finite multiverses, and the relationship persists in the limit $n_{\text {tot }} \rightarrow \infty$.)

Analogous results are obtained for regions of type $I$. We simply need to replace $F$ with $I$ and $\kappa_{F F}$ by $\kappa_{I I}$ in Eq. (75).

The "Boltzmann brains" are created at a very small rate $\kappa_{j}^{B B}$ per $H$-region of type $j$, while ordinary observers are created at a much larger rate per reheated 3 -volume $H_{j}^{-3}$ in bubbles of the same type. We conclude that the RV-regulated abundance of "Boltzmann brains" is always negligible compared with the abundance of ordinary observers in bubbles of the same type.

\section{A GENERAL LANDSCAPE}

In the previous section we performed computations in a simple toy model of the landscape. Let us now consider a general landscape containing $N$ vacua, labeled $j=1$, ..., $N$. The dimensionless transition rates $\kappa_{j \rightarrow k}$ between vacua $j$ and $k$ are considered known. The main task is to compute the RV-regulated ratio of abundances of bubbles of kinds $j$ and $k$. We will also compare the abundances of ordinary observers with that of Boltzmann brains.

\section{A. Bubble abundances}

It is convenient to denote by $T$ the set of terminal bubble types and to relabel the vacua such that $j=1$, ..., $N_{r}$ are the "recyclable" (nonterminal) vacua. Thus, $T=\left\{N_{r}+1, \ldots, N\right\}$. We start by considering the probability $p\left(n,\left\{n_{j}\right\} ; k\right)$ of having $n_{j}$ bubbles of type $j$, with total $n=\sum_{j} n_{j}$ bubbles of all types, to the future of an initial bubble of type $k \notin T$. The generating function $g\left(z,\left\{q_{j}\right\} ; k\right)$ for this probability distribution can be defined by a straightforward generalization of Eq. (7),

$$
g\left(z,\left\{q_{l}\right\} ; k\right) \equiv \sum_{n,\left\{n_{l}\right\} \geq 0} p\left(n,\left\{n_{l}\right\} ; k\right) z^{n} \prod_{j} q_{j}^{n_{j}} .
$$

Here $z$ is the generating parameter for $n$, and $q_{j}$ are the generating parameters for $n_{j}$. The generating function can be written symbolically as the average

$$
g\left(z,\left\{q_{l}\right\} ; k\right) \equiv\left\langle z^{n} q_{1}^{n_{1}} \ldots q_{N}^{n_{N}}\right\rangle_{n<\infty ; k},
$$

where the subscript $(n<\infty)$ indicates that only the events with a finite total number of bubbles contribute to the statistical average.

\footnotetext{
3 Typically $F_{*} \ll 1$ but we can do with a weaker bound $F_{*}<1$ here.
} 
For terminal bubble types $k \in T$, the definition (76) yields $g\left(z,\left\{q_{j}\right\} ; k\right)=1$ since there are no further bubbles to the future of terminal bubbles, thus $n=n_{j}=0$ with probability 1 .

The generating function $g\left(z,\left\{q_{j}\right\} ; k\right), k=1, \ldots, N_{r}$ satisfies the system of $N_{r}$ nonlinear equations

$$
\begin{aligned}
g^{\frac{1}{\nu}}\left(z,\left\{q_{j}\right\} ; k\right)= & \sum_{i \notin T, i \neq k} z q_{i} \kappa_{k \rightarrow i} g\left(z,\left\{q_{j}\right\} ; i\right) \\
& +\sum_{i \in T} z q_{i} \kappa_{k \rightarrow i}+\kappa_{k \rightarrow k} g\left(z,\left\{q_{j}\right\} ; k\right),
\end{aligned}
$$

where $\nu \equiv e^{3}$ as before, and the quantities $\kappa_{k \rightarrow k}$ defined by Eq. (6). A derivation of Eqs. (78) will be given below in Sec. IVD.

The solution of Eqs. (78) can be visualized as $N_{r}$ analytic functions $g\left(z,\left\{q_{j}\right\} ; k\right), k=1, \ldots, N_{r}$, of the free parameters $z$ and $q_{j}(j=1, \ldots, N)$. Arguments similar to those of Sec. III show that the physically significant solution of Eqs. (78) is the "main branch" that has the following asymptotic form at $z \rightarrow 0$,

$$
g(z ; k)=z^{\nu}\left[\sum_{i \in T} q_{i} \kappa_{k \rightarrow i}\right]^{\nu}+O\left(z^{2 \nu-1}\right)
$$

(see also the argument at the end of Sec. IVD).

Once the generating function $g\left(z,\left\{q_{j}\right\} ; k\right)$ is determined, the RV measure gives the ratio of the mean number of bubbles of type $j$ to that of type $k$ as

$$
\frac{p(j)}{p(k)}=\left.\lim _{n \rightarrow \infty} \frac{\partial_{z}^{n} \partial_{q_{j}} g\left(z,\left\{q_{i}\right\} ; i^{\prime}\right)}{\partial_{z}^{n} \partial_{q_{k}} g\left(z,\left\{q_{i}\right\} ; i^{\prime}\right)}\right|_{z=0,\left\{q_{i}\right\}=1},
$$

where, for clarity, we wrote explicitly the type $i^{\prime}$ of the initial bubble. We will now use the methods developed in Sec. IIIA to reduce Eq. (80) to an expression that does not contain limits and so can be analyzed more easily. It will then become evident that the limit (80) is independent of $i^{\prime}$.

For a fixed bubble type $j$, we first consider the derivatives $\partial_{q_{j}} g\left(z,\left\{q_{i}\right\} ; k\right), k=1, \ldots, N_{r}$, evaluated at $\left\{q_{i}\right\}=1$. Let us denote these $N_{r}$ derivatives by $h_{j}(z ; k)$,

$$
\left.h_{j}(z ; k) \equiv \frac{\partial}{\partial q_{j}}\right|_{q_{i}=1} g\left(z,\left\{q_{i}\right\} ; k\right), \quad k=1, \ldots, N_{r} .
$$

These quantities are conveniently represented by an $N_{r^{-}}$ dimensional vector, which we will denote by $\left|h_{j}(z)\right\rangle$ using the Dirac notation (although no connection to quantum mechanics is present here). This vector satisfies an inhomogeneous linear equation that follows straightforwardly by taking the derivative $\partial_{q_{j}}$ at $\left\{q_{i}\right\}=1$ of Eqs. (78). That equation can be written in the matrix form as follows,

$$
\begin{aligned}
& \sum_{i=1}^{N} M_{k i}(z) h_{j}(z ; i)=z \kappa_{k \rightarrow j} g(z ; j), \quad k \neq j, \\
& \sum_{i=1}^{N} M_{k i}(z) g_{, q_{j}}(z ; i)=0, \quad k=j,
\end{aligned}
$$

where we denoted by $g(z ; j) \equiv g\left(z ;\left\{q_{i}\right\}=1 ; j\right)$ the solution of Eqs. (78) at $\left\{q_{i}\right\}=1$, written as

$$
\begin{aligned}
g^{\frac{1}{\nu}}(z ; k)= & z \sum_{i \notin T, i \neq k} \kappa_{k \rightarrow i} g(z ; i) \\
& +z \sum_{i \in T} \kappa_{k \rightarrow i}+\kappa_{k \rightarrow k} g(z ; k),
\end{aligned}
$$

while the matrix $M_{k i}(z)$ is defined by

$$
\hat{M}(z) \equiv M_{k i}(z)= \begin{cases}\frac{1}{\nu} g^{\frac{1}{\nu}-1}(z ; k)-\kappa_{k \rightarrow k}, & k=i \\ -z \kappa_{k \rightarrow i}, & k \neq i .\end{cases}
$$

For convenience we rewrite Eqs. (82) - in a more concise form,

$$
\hat{M}(z)\left|h_{j}(z)\right\rangle=\left|Q_{j}(z)\right\rangle,
$$

where $\left|Q_{j}\right\rangle$ is the vector with the components $\left|Q_{j}\right\rangle_{i}, i=$ $1, \ldots, N_{r}$ given by the right-hand sides of Eqs. (82)-

$$
\left|Q_{j}(z)\right\rangle_{i} \equiv z \kappa_{i \rightarrow j} g(z ; j)\left[1-\delta_{i j}\right] .
$$

The solution of Eq. (86) is found symbolically as

$$
\left|h_{j}(z)\right\rangle=\hat{M}^{-1}(z)\left|Q_{j}(z)\right\rangle
$$

provided that the inverse matrix $\hat{M}^{-1}(z)$ exists. Of course, it is impractical to obtain the inverse matrix explicitly; but we will never need to do that.

The next task is to determine $z$ for which the matrix $\hat{M}(z)$ remains nondegenerate, $\operatorname{det} \hat{M}(z) \neq 0$. It will be shown in Sec. IVE that there exists an eigenvalue $\lambda_{0}(z)$ of $\hat{M}(z)$ such that $\lambda_{0}\left(z_{*}\right)=0$ at some real $z_{*}>0$, while $\lambda_{0}(z)>0$ for $z<z_{*}$. Moreover, the (left and right) eigenvectors corresponding to the eigenvalue $\lambda_{0}(z)$ are always nondegenerate and can be chosen with all positive components. At the same time, all the other eigenvalues of $\hat{M}(z)$ remain nonzero for all $z \leq z_{*}$. It will also be shown (see Sec. IVF) that the following asymptotic expansion holds near $z=z_{*}$,

$$
\lambda_{0}(z)=c_{1} \sqrt{z_{*}-z}+O\left(z_{*}-z\right), \quad c_{1}>0 .
$$

One can see from the small- $z$ asymptotics (79) and from Eq. (85) that, for small enough $z, \hat{M}(z)$ contains very large positive numbers on the diagonal and very small negative numbers off the diagonal. Hence $\operatorname{det} \hat{M}(z)>0$ for all sufficiently small $z$. Since the eigenvalues of $\hat{M}(z)$ remain nonzero except for $\lambda_{0}(z)$ that first vanishes at $z=z_{*}$, we conclude that $\operatorname{det} \hat{M}(z)$ remains positive for all $0<z<z_{*}$, and that $z=z_{*}$ is the smallest positive value of $z$ for which $\operatorname{det} \hat{M}(z)=0$.

Now we restrict our attention to $0<z<z_{*}$, for which $\operatorname{det} \hat{M}(z)>0$ and $\hat{M}^{-1}(z)$ exists. We need to analyze the behavior of $\hat{M}^{-1}$ near $z \approx z_{*}$. We treat $\hat{M}(z)$ as an operator in $N_{r}$-dimensional real space $V$. The matrix $\hat{M}$ is not symmetric and may not be diagonalizable. Instead of diagonalizing $\hat{M}(z)$, we split the space $V$ into 
the 1-dimensional subspace corresponding to the smallest eigenvalue $\lambda_{0}(z)$, and into the $\left(N_{r}-1\right)$-dimensional complement subspace $V_{1}$. We know that the eigenvalue $\lambda_{0}(z)$ is nondegenerate. Thus, we may symbolically write

$$
\hat{M}(z)=\lambda_{0}(z)\left|v^{0}(z)\right\rangle\left\langle u^{0}(z)\right|+\hat{M}_{1}(z),
$$

where $\left|v^{0}\right\rangle$ and $\left\langle u^{0}\right|$ are the right and the left eigenvectors corresponding to $\lambda_{0}(z)$, normalized such that

$$
\left\langle u^{0} \mid v^{0}\right\rangle=1
$$

and it is implied that $\hat{M}_{1}(z)$ vanishes on $\left|v^{0}\right\rangle$ and $\left\langle u^{0}\right|$ but is nonsingular on the complement space $V_{1}$. In other words, we have

$$
\begin{aligned}
\hat{M}\left|v^{0}\right\rangle & =\lambda_{0}\left|v_{0}\right\rangle, \quad\left\langle u^{0}\right| \hat{M}=\lambda_{0}\left\langle u^{0}\right|, \\
\hat{M}_{1}\left|v^{0}\right\rangle & =0, \quad\left\langle u^{0}\right| \hat{M}_{1}=0 .
\end{aligned}
$$

There exists a matrix $\hat{M}_{1\left(V_{1}\right)}^{-1}$ that acts as the inverse to $\hat{M}_{1}$ when restricted to the subspace $V_{1}$ and again vanishes on $\left|v^{0}\right\rangle$ and $\left\langle u^{0}\right|$. Using that matrix, we may write the inverse matrix $\hat{M}^{-1}$ explicitly as

$$
\hat{M}^{-1}(z)=\frac{1}{\lambda_{0}(z)}\left|v^{0}(z)\right\rangle\left\langle u^{0}(z)\right|+\hat{M}_{1\left(V_{1}\right)}^{-1}(z) .
$$

Hence, the solution (88) can be written as

$$
\left|h_{j}(z)\right\rangle=\frac{\left\langle u^{0} \mid Q_{j}\right\rangle}{\lambda_{0}(z)}\left|v^{0}\right\rangle+\hat{M}_{1\left(V_{1}\right)}^{-1}\left|Q_{j}\right\rangle .
$$

Now it is clear from Eq. (89) that $\left|h_{j}(z)\right\rangle$ diverges at $z=z_{*}$ as $\propto\left(z_{*}-z\right)^{-1 / 2}$. It also follows that $\left|h_{j}(z)\right\rangle$ does not diverge at any smaller real $z$.

We can also show that $\lambda_{0}(z)$ cannot vanish at some complex value of $z$ that is closer to $z=0$ than $z=z_{*}$. If $\lambda_{0}\left(z_{*}^{\prime}\right)=0$ with a complex-valued $z_{*}^{\prime}$, then a derivative of the generating function, such as $\partial_{z} g(z ; j)$, would diverge at $z=z_{*}^{\prime}$. Using the definition (76) of $g$ and substituting $q_{i}=1$, we find that the following sum diverges,

$$
\left.\frac{\partial g(z ; j)}{\partial z}\right|_{z=z_{*}^{\prime}}=\sum_{n \geq 1}\left(z_{*}^{\prime}\right)^{n-1} n p(n ; j)=\infty .
$$

The sum of absolute values is not smaller than the above, and hence also diverges:

$$
\left.\frac{\partial g(z ; j)}{\partial z}\right|_{z=\left|z_{*}^{\prime}\right|}=\sum_{n \geq 1}\left|z_{*}^{\prime}\right|^{n-1} n p(n ; j)=\infty .
$$

So $\partial_{z} g$ has also a singularity at a real value $z=\left|z_{*}^{\prime}\right|$. As we have shown, $z=z_{*}$ is the smallest such real-valued singularity point; hence $\left|z_{*}^{\prime}\right| \geq z_{*}$. It follows that $z_{*}$ is equal to the radius of convergence of the series (96), which is a Taylor series for the function $\partial_{z} g$. There remains the possibility that a singularity $z_{*}^{\prime}$ is located directly on the circle of convergence, so that $\left|z_{*}^{\prime}\right|=z_{*}$ and $z_{*}^{\prime}=z_{*} e^{\mathrm{i} \phi}$ with $0<\phi<2 \pi$. This possibility can be excluded using the following argument. The function $\partial_{z} g$ can have only finitely many singularities on the circle $|z|=z_{*}$; infinitely many singularities on the circle would indicate an accumulation point which would be an essential singularity, i.e. not a branch point. However, by construction $g(z ; j)$ is an algebraic function of $z$ that cannot have singularities other than branch points. Since $g(z ; j)$ is regular at $z=z_{*}^{\prime}$, all branch points of $g$ must be of the form $\left(z-z_{*}^{\prime}\right)^{s}$ with $s>0$. Using the explicit formula (25) for the $n$-th derivative of an analytic function with a branch cut singularity of the form $\left(z-z_{*}^{\prime}\right)^{s}$, we find that the coefficients $n p(n ; j)$ of the Taylor series (96) decay at large $n$ asymptotically as

$$
\begin{aligned}
n p(n ; j) & =\frac{1}{n !} \partial_{z}^{n} \partial_{z} g \propto \frac{\Gamma(n+1-s)}{\Gamma(n+1)}\left(z_{*}^{\prime}\right)^{-n}\left(1+O\left(n^{-1}\right)\right) \\
& \propto n^{-s}\left(z_{*}^{\prime}\right)^{-n}\left(1+O\left(n^{-1}\right)\right) .
\end{aligned}
$$

The function $g$ has a finite number of singularities $z_{*}^{\prime}=$ $z_{*} e^{\mathrm{i} \phi}$ on the circle $|z|=z_{*}$, and each singularity gives a contribution of the form (98). Hence, we may estimate (for sufficiently large $n$, say for $n \geq n_{0}$ )

$$
n p(n ; j)=c_{0} n^{-s} z_{*}^{-n}\left(1+O\left(n^{-1}\right)\right), \quad c_{0}>0 .
$$

Then the partial sum for $n \geq n_{0}$ of the series (96) is estimated by

$$
\begin{aligned}
\sum_{n \geq n_{0}}\left(z_{*}^{\prime}\right)^{n-1} n p(n ; j) & \approx \sum_{n \geq n_{0}}\left(z_{*}^{\prime}\right)^{n-1} c_{0} n^{-s} z_{*}^{-n} \\
& =\frac{c_{0}}{z_{*}} \sum_{n \geq n_{0}} n^{-s} e^{\mathrm{i} n \phi} .
\end{aligned}
$$

The latter series converges for $s>0$ and $0<\phi<2 \pi$. (The neglected terms of order $n^{-1-s}$ build an absolutely convergent series and hence introduce an arbitrarily small error into the estimate.) Therefore, the series (96) also converges at $z_{*}^{\prime} \neq z_{*}$, contradicting the assumption that other singularities exist on the circle $|z|=z_{*}$.

We conclude from these arguments that the singularity of $g(z ; j)$ nearest to $z=0$ in the complex $z$ plane is indeed at a real value $z=z_{*}$, and all other singular points $z_{*}^{\prime}$ satisfy the strict inequality $\left|z_{*}^{\prime}\right|>z_{*}$. The same statement about the locations of singularities holds for the generating functions $g\left(z,\left\{q_{i}\right\} ; j\right)$ and hence for their derivatives such as $\left|h_{j}(z)\right\rangle$.

To compute the final expression (80), we need to evaluate the $n$-th derivative $\partial_{z}^{n}$ of the functions $h_{j}(z ; i)$ at $z=0$ and for very large $n$. To this end, we use the formula (25), which requires to know the location $z=z_{*}$ of the singularity of $h_{j}(z ; i)$ nearest to $z=0$. We have just found that this singularity is at a real value $z=z_{*}$ and has the form

$$
h_{j}(z ; i) \approx \frac{1}{c_{1} \sqrt{z_{*}-z}}\left\langle u^{0} \mid Q_{j}\right\rangle v_{i}^{0}, \quad z \approx z_{*},
$$

where $v_{i}^{0}$ is the $i$-th component of the eigenvector $\left|v_{0}\right\rangle$. The value of the proportionality constant $c_{1}$ is not required for computing the ratios (80). Using Eqs. (25), 
(87), and (101), we evaluate the limit (80) as

$$
\frac{p(j)}{p(k)}=\left.\frac{\left\langle u^{0} \mid Q_{j}\right\rangle v_{i^{\prime}}^{0}}{\left\langle u^{0} \mid Q_{k}\right\rangle v_{i^{\prime}}^{0}}\right|_{z=z_{*}}=\frac{\sum_{i \neq j} u_{i}^{0}\left(z_{*}\right) \kappa_{i \rightarrow j}}{\sum_{i \neq k} u_{i}^{0}\left(z_{*}\right) \kappa_{i \rightarrow k}} \frac{g\left(z_{*} ; j\right)}{g\left(z_{*} ; k\right)} .
$$

Since the component $v_{i^{\prime}}^{0}$ cancels, we find that the limit (80) is indeed independent of the initial bubble type $i^{\prime}$. We also note that the normalization of the eigenvector $\left\langle u^{0}\right|$ is irrelevant for the ratio.

The formula (102) can be simplified further for nonterminal types $j, k$ if we use the relationship $\left\langle u^{0}\left(z_{*}\right)\right| \hat{M}\left(z_{*}\right)=0$ together with the explicit definition (85) of $\hat{M}$ :

$$
\begin{aligned}
0 & =\sum_{i \neq j} u_{i}^{0}\left(z_{*}\right) M_{i j}\left(z_{*}\right)+u_{j}^{0}\left(z_{*}\right) M_{j j}\left(z_{*}\right) \\
& =-\sum_{i \neq j} u_{i}^{0}\left(z_{*}\right) z_{*} \kappa_{i \rightarrow j}+u_{j}^{0}\left(z_{*}\right)\left[\frac{1}{\nu} g^{\frac{1}{\nu}-1}\left(z_{*} ; j\right)-\kappa_{j \rightarrow j}\right] .
\end{aligned}
$$

The last term in the square brackets in Eq. (103) can be neglected since $\kappa_{j \rightarrow j} \approx 1$ while $g\left(z_{*} ; j\right) \ll 1$. Hence, Eq. (102) is simplified to

$$
\frac{p(j)}{p(k)} \approx \frac{u_{j}^{0}\left(z_{*}\right)}{u_{k}^{0}\left(z_{*}\right)}\left[\frac{g\left(z_{*} ; j\right)}{g\left(z_{*} ; k\right)}\right]^{1 / \nu} .
$$

This is the main formula for the RV-regulated relative abundances of bubbles of arbitrary (nonterminal) types $j$ and $k$. For a terminal type $k \in T$, one needs to use Eq. (102) together with $g(z ; k) \equiv 1$.

We note that the expression (104) depends on the components $u_{i}^{0}$ of the left eigenvector of the matrix $\hat{M}\left(z_{*}\right)$ with eigenvalue $\lambda_{0}\left(z_{*}\right)=0$. Although we have been able to evaluate the limit (80) analytically and obtained Eq. (104), the task of computing the values of $z_{*}, g\left(z_{*} ; j\right)$, and $u_{i}^{0}\left(z_{*}\right)$ remains quite difficult. The outline of the required computations is as follows: One first needs to determine $g(z ; j)\left(j=1, \ldots, N_{r}\right)$ as the "main branch" of the solution of Eqs. (84) with the small- $z$ asymptotic given by Eq. (79). The functions $g(z ; j)$ determine the matrix elements $M_{i j}(z)$ using Eq. (85). Then one needs to find the smallest value $z=z_{*}>0$ such that the determinant of the matrix $\hat{M}(z)$ vanishes. Finally, one needs to compute a left eigenvector $\left\langle u^{0}\left(z_{*}\right)\right|$ that corresponds to the eigenvalue $\lambda_{0}\left(z_{*}\right)=0$ of the matrix $\hat{M}\left(z_{*}\right)$. The mathematical construction shown below guarantees that $z_{*}$ exists and that $\left\langle u^{0}\left(z_{*}\right)\right|$ is nondegenerate and has all positive components; the results are independent of the normalization of $\left\langle u^{0}\right|$. However, a brute-force numerical computation of these quantities appears to be impossible due to the huge number $N_{r}$ of the simultaneous equations (84) and to the wide range of numerical values of the coefficients $\kappa_{i \rightarrow j}$ in a typical landscape. Even the numerical value of $z_{*}$ is likely to be too large to be represented efficiently in computers. In the next section we will consider an example landscape where an analytic approximation can be found.

\section{B. Example landscape}

We begin with some qualitative considerations regarding the behavior of the functions $g(z ; k)$.

To determine $g\left(z_{*} ; k\right)$, we need to follow the main branch $g(z ; k)$ as the value of $z$ is increased from $z=0$ until $\operatorname{det} \hat{M}(z)$ vanishes, which will determine the value $z=z_{*}$. We note that the asymptotic form (79) is valid in some range near $z=0$. Since $g(z ; k) \ll 1$ at those $z$, the matrix $\hat{M}(z)$ is dominated by large positive diagonal terms $g^{\frac{1}{\nu}-1}(z ; k) \delta_{k j}$. As $z$ increases to $z_{*}$, all the functions $g(z ; j)$ also increase while $\operatorname{det} \hat{M}(z)$ decreases monotonically to zero (this statement is proved rigorously in Sec. IVE). One can visualize the changes in the matrix elements of $\hat{M}(z)$ if one notes that the positive diagonal terms decrease with $z$ while the negative off-diagonal terms $\left(M_{j k}=-z \kappa_{j \rightarrow k}, j \neq k\right)$ grow in magnitude. Eventually $\operatorname{det} \hat{M}(z)$ vanishes at $z=z_{*}$ such that the off-diagonal terms become sufficiently large at least in some rows and columns of the matrix $\hat{M}(z)$.

Let us determine an upper bound on $z$ such that Eq. (79) remains a good approximation for the generating function $g(z ; j) \equiv g\left(z,\left\{q_{i}=1\right\} ; j\right)$. This will be the case if the term $z \sum_{i \in T} \kappa_{k \rightarrow i}$ in the right hand side of Eq. (84) dominates over all other terms, for every $k$. Denoting for brevity

$$
\kappa_{k \rightarrow T} \equiv \sum_{i \in T} \kappa_{k \rightarrow i}
$$

the total transition probability from $k$ to all terminal vacua, we have therefore the conditions (for every $k$ )

$$
z \kappa_{k \rightarrow T} \gg g(z ; k), \quad z \kappa_{k \rightarrow T} \gg z \sum_{i \notin T, i \neq k} \kappa_{k \rightarrow i} g(z ; i) .
$$

These conditions are satisfied, consistently with Eq. (79), if $z$ is bounded (for every $k$ ) simultaneously by

$$
z \ll \kappa_{k \rightarrow T}^{-1}, \quad z \ll\left[\sum_{i \notin T, i \neq k} \frac{\kappa_{k \rightarrow i}}{\kappa_{k \rightarrow T}} \kappa_{i \rightarrow T}^{\nu}\right]^{-\frac{1}{\nu}} .
$$

Since typically the rate of transitions to terminal vacua is much smaller than the rate of transitions to $\mathrm{dS}$ vacua, one can expect that this regime will include $z=1$. Nevertheless, one expects that $\operatorname{det} \hat{M}(z)$ remains nonzero for these $z$. The detailed consideration of the FABI model in Sec. IIIA showed that the value of $z_{*}$ lies outside the regime (107) unless the landscape parameters are finetuned. In a general landscape, one expects the analogous fine-tuning to be much stronger or even impossible to satisfy. In other words, one expects that the functions $g\left(z_{*} ; k\right)$ at least for some $k$ will violate the conditions (106), although these conditions might be satisfied for other $k$.

Now we would like to estimate the value of $z$ for which the matrix $\hat{M}(z)$ first becomes degenerate. We can use a 
general theorem for estimating the eigenvalues of a matrix (Gershgorin's theorem, see [59], chapter 7). For each of the diagonal elements $M_{k k}, k=1, \ldots, N_{r}$ one needs to draw a circle in the complex $\lambda$ plane, centered at the diagonal element $M_{k k}$ with radius

$$
\rho_{k} \equiv \sum_{i \neq k}\left|M_{k i}\right|
$$

Thus one obtains $N_{r}$ circles

$$
\left|\lambda-M_{k k}\right| \leq \rho_{k}, \quad k=1, \ldots, N_{r},
$$

called the Gershgorin circles. The Gershgorin theorem says that all the eigenvalues of a matrix $M_{j k}$ are located within the union of these circles. An elementary proof is as follows. An eigenvector $v_{i}$ with eigenvalue $\lambda$ can be normalized such that its component of largest absolute value is equal to 1 . Let $v_{1}=1$ be this component (renumbering the components if necessary), then it is easy to show that $\lambda$ is within a circle with center $M_{11}$ and radius $\rho_{1}$. Namely, we start from the eigenvalue equation,

$$
\sum_{i \neq 1} M_{1 i} v_{i}+M_{11} v_{1}=\lambda v_{1}
$$

and use the properties $v_{1}=1$ and $\left|v_{i}\right| \leq 1$ for $i \neq 1$ :

$$
\left|\lambda-M_{11}\right|=\left|\sum_{i \neq 1} M_{1 i} v_{i}\right| \leq \sum_{i \neq 1}\left|M_{1 i}\right|=\rho_{1} .
$$

Applying the theorem to the matrix $\hat{M}(z)$ at small $z$ such that Eq. (79) is a good approximation for the generating function $g(z ; j)$, we find that the Gershgorin circles are centered at large positive values

$$
M_{k k}=\frac{1}{\nu} g^{\frac{1}{\nu}-1}(z ; k)-\kappa_{k \rightarrow k} \approx \frac{1}{\nu} \frac{1}{\left(z \kappa_{k \rightarrow T}\right)^{\nu-1}} \gg 1,
$$

while the radii of the circles are small,

$$
\rho_{k}=z \sum_{i \neq k, i \notin T} \kappa_{k \rightarrow i} \ll 1
$$

Hence, for small $z$ none of the circles can contain $\lambda=0$, so the matrix $\hat{M}(z)$ is nondegenerate. As $z$ increases, the radii $\rho_{k}$ increase while the centers $M_{k k}$ move monotonically towards zero. The circle closest to $\lambda=0$ is the one with the center closest to zero and the largest radius. This circle is labeled by $k$ with largest rates $\kappa_{k \rightarrow T}$ and $\kappa_{k \rightarrow i}$. This value of $k$ corresponds to the highenergy vacua in the landscape, for which tunneling to any other vacua is much easier than for low-energy vacua. Therefore the eigenvalue $\lambda\left(z_{*}\right)=0$ will be located inside the Gershgorin circle(s) centered at $M_{k k}\left(z_{*}\right)$ for $k$ corresponding to high-energy vacua. The Gershgorin circle to which the eigenvalue belongs indicates the largest component of the eigenvector. Hence, one expects that the eigenvector $\left\langle u^{0}\left(z_{*}\right)\right|$ has the largest values of its components $u_{k}^{0}$ corresponding to the high-energy vacua $k$. The Gershgorin theorem requires that $\lambda_{0}\left(z_{*}\right)=0$ be inside the circle centered at $M_{k k}\left(z_{*}\right)$ with radius $\rho_{k}\left(z_{*}\right)$, i.e.

$$
M_{k k}\left(z_{*}\right)=\frac{1}{\nu} g^{\frac{1}{\nu}-1}\left(z_{*} ; k\right)-\kappa_{k \rightarrow k} \leq z_{*} \sum_{i \neq k, i \notin T} \kappa_{k \rightarrow i}
$$

Disregarding the terms $\kappa_{k \rightarrow k}$, which are small in comparison with the remaining terms, and using Eq. (84) for $g\left(z_{*} ; k\right)$ we obtain the following condition,

$$
z_{*}^{\nu} \geq \frac{1}{\nu} \frac{\left[\kappa_{k \rightarrow T}+\sum_{i \neq k, i \notin T} \kappa_{k \rightarrow i} g\left(z_{*} ; i\right)\right]^{1-\nu}}{\sum_{i \neq k, i \notin T} \kappa_{k \rightarrow i}} .
$$

Proceeding further requires at least an estimate of $g\left(z_{*} ; i\right)$ for all $i$, which is so far not available.

While we are as yet unable to obtain an explicit estimate of $z_{*}$ and $\left\langle u^{0}\right|$ for an arbitrary landscape, let us consider a workable example that is more realistic than the FABI model. In this example there is a single highenergy vacuum, labeled $k=1$, and a large number of dS low-energy vacua, $k=2, \ldots, N_{r}$, as well as a number of terminal vacua, $k=N_{r}+1, \ldots, N$. We assume that the downward tunneling rates $\kappa_{1 \rightarrow i}$ for $i \neq 1$ are much larger than the rates $\kappa_{i \rightarrow 1}$ or $\kappa_{i \rightarrow j}$ for $i, j \neq 1$. Then $g(z ; 1) \gg g(z ; i)$ for $i \neq 1$, and so the first Gershgorin circle is the one closest to $\lambda=0$. Hence we may expect that the eigenvalue $\lambda_{0}(z)$ always belongs to that circle. Moreover, it is likely that $z_{*}$ is within the regime (106) for the low-energy vacua $(k \neq 1)$ but not for the highenergy vacuum $k=1$. We will now proceed with the calculation and later check that these assumptions are self-consistent.

If the regime (106) holds for every low-energy vacuum $k \neq 1$, we have

$$
g(z ; k) \approx z^{\nu} \kappa_{k \rightarrow T}^{\nu}, \quad k=2, \ldots, N_{r} .
$$

For the high-energy vacuum $k=1$ we use Eq. (84) directly to find

$$
\begin{aligned}
g(z ; 1) & =\left[\kappa_{1 \rightarrow T}+z \sum_{i \notin T, i \neq 1} \kappa_{1 \rightarrow i} g(z ; i)+\kappa_{1 \rightarrow 1} g(z ; 1)\right]^{\nu} \\
& \approx z^{\nu^{2}+\nu}\left[\sum_{i=2}^{N_{r}} \kappa_{1 \rightarrow i} \kappa_{i \rightarrow T}^{\nu}\right]^{\nu},
\end{aligned}
$$

where we have neglected the terms with $\kappa_{1 \rightarrow T}$ and $\kappa_{1 \rightarrow 1}$. The condition that the regime (106) holds for $k \neq 1$ is

$$
\kappa_{i \rightarrow T} \gg z \sum_{j \neq i, j \notin T} \kappa_{i \rightarrow j} g(z ; j), \quad i=2, \ldots, N_{r}
$$

In the matrix $\hat{M}(z)$ the off-diagonal elements $M_{i j}$ for $i \neq 1, j \neq 1$ are much smaller than all other elements. 
Hence, we can approximate $\hat{M}(z)$ by a matrix that has only its first row, first column, and the diagonal elements,

$$
\hat{M}(z) \approx\left(\begin{array}{ccccc}
M_{11} & -z \kappa_{1 \rightarrow 2} & -z \kappa_{1 \rightarrow 3} & \cdots & -z \kappa_{1 \rightarrow N_{r}} \\
-z \kappa_{2 \rightarrow 1} & M_{22} & 0 & \cdots & 0 \\
\vdots & 0 & \ddots & & \vdots \\
-z \kappa_{N_{r} \rightarrow 1} & 0 & 0 & \cdots & M_{N_{r} N_{r}}
\end{array}\right),
$$$$
M_{k k} \approx \frac{1}{\nu} g^{\frac{1}{\nu}-1}(z ; k) \text {. }
$$

This approximate matrix is much easier to analyze; in particular, its determinant and the eigenvectors can be computed in closed form. The determinant of this matrix is

$$
\operatorname{det} \hat{M}(z) \approx M_{11} \ldots M_{N_{r} N_{r}}\left[1-\sum_{i=2}^{N_{r}} \frac{z^{2} \kappa_{1 \rightarrow i} \kappa_{i \rightarrow 1}}{M_{11}(z) M_{i i}(z)}\right] .
$$

Therefore, the condition $\operatorname{det} \hat{M}\left(z_{*}\right)=0$ can be written as

$$
M_{11}\left(z_{*}\right) \approx z_{*}^{2} \sum_{i=2}^{N_{r}} \frac{\kappa_{1 \rightarrow i} \kappa_{i \rightarrow 1}}{M_{i i}\left(z_{*}\right)} .
$$

Using Eqs. (116), (117), and (119), we transform this condition to

$$
z_{*}^{-\nu-\nu^{2}} \approx \nu^{2}\left[\sum_{i=2}^{N_{r}} \kappa_{1 \rightarrow i} \kappa_{i \rightarrow T}^{\nu}\right]^{\nu-1} \sum_{i=2}^{N_{r}} \kappa_{1 \rightarrow i} \kappa_{i \rightarrow 1} \kappa_{i \rightarrow T}^{\nu-1} .
$$

However, one does not need this value apart from checking explicitly that the assumptions (118) hold.

The left eigenvector $\left\langle u^{0}\left(z_{*}\right)\right|$ corresponding to the eigenvalue 0 of the matrix $\hat{M}\left(z_{*}\right)$ is found approximately as

$$
u_{1}^{0}=1, \quad u_{k}^{0} \approx \frac{z_{*} \kappa_{1 \rightarrow k}}{M_{k k}\left(z_{*}\right)},
$$

where the normalization $u_{1}^{0}=1$ was chosen arbitrarily for convenience. A perturbative improvement of this approximation along the lines of Refs. [44, 47, 48, 49] may be possible, but the precision obtained from the present approximation is sufficient for our purposes.

The bubble abundance ratios (104) for nonterminal types are then expressed as follows,

$$
\begin{aligned}
& \frac{p(j)}{p(k)} \approx \frac{\kappa_{1 \rightarrow j}}{\kappa_{1 \rightarrow k}} \frac{\kappa_{j \rightarrow T}^{\nu}}{k_{k \rightarrow T}^{\nu}}, \quad j, k=2, \ldots, N_{r}, \\
& \frac{p(1)}{p(k)} \approx \frac{1}{\nu} \frac{\sum_{i=2}^{N_{r}} \kappa_{1 \rightarrow i} \kappa_{i \rightarrow T}^{\nu}}{\kappa_{1 \rightarrow k} \kappa_{k \rightarrow T}^{\nu}}, \quad k=2, \ldots, N_{r} .
\end{aligned}
$$

These equations are the main result of this section, yielding RV-regulated bubble abundances for a landscape with a large number of low-energy vacua. The formula (125) agrees with that obtained in Sec. IIIA for the ratio $p(F): p(I) \approx 1 / \nu$ in the FABI landscape, which may be considered a special case of the present model with $N_{r}=2$.

\section{C. "Boltzmann brains"}

We now investigate the abundance of "Boltzmann brains" in a general landscape, relative to the abundance of ordinary observers.

We first need to derive the equations for the suitable generating functions, analogously to Eqs. (70)-(71). Let us introduce the generating function

$$
g\left(z,\left\{q_{i}\right\},\left\{r_{i}\right\} ; j\right) \equiv\left\langle z^{n_{\mathrm{tot}}} \prod_{i} q_{i}^{n_{i}} r_{i}^{N_{i}}\right\rangle_{n_{\mathrm{tot}}<\infty ; j},
$$

where $n_{i}$ is the total number of bubbles of type $i$ and $N_{i}$ is the total number of $H$-regions of type $i$. The number of BBs in bubbles of type $i$ is proportional to $N_{i}$ with a proportionality constant $\kappa_{i}^{B B}$, which is the (extremely small) nucleation rate of a $\mathrm{BB}$ per Hubble 4-volume $H_{i}^{-4}$.

The generating function $g\left(z,\left\{q_{i}\right\},\left\{r_{i}\right\} ; j\right)$, which we will denote for brevity by $g(\ldots, j)$, satisfies a system of equations analogous to Eq. (78),

$$
g^{\frac{1}{\nu}}(\ldots, j)=\sum_{k \neq j} \kappa_{j \rightarrow k} z q_{k} r_{k} g(\ldots, k)+\kappa_{j \rightarrow j} r_{j} g(\ldots, j) .
$$

Let us compute the RV-regulated ratio of the number of $H$-regions of type $j$ to the number of bubbles of the same type $j$. This ratio is given by

$$
\lim _{n \rightarrow \infty} \frac{\left\langle N_{j}\right\rangle_{n}}{\left\langle n_{j}\right\rangle_{n}}=\left.\lim _{n \rightarrow \infty} \frac{\partial_{z}^{n} \partial_{r_{j}} g\left(\ldots, i^{\prime}\right)}{\partial_{z}^{n} \partial_{q_{j}} g\left(\ldots, i^{\prime}\right)}\right|_{z=0, r_{i}=1, q_{i}=1},
$$

where $i^{\prime}$ is the initial bubble type. To evaluate the limit, we use the methods developed in Sec. IVA. The derivatives $\partial_{q_{j}} g \equiv\left|h_{j}(z)\right\rangle$, as defined in Eq. (81), satisfy Eq. (86). The vector of derivatives

$$
\left.\frac{\partial}{\partial r_{j}}\right|_{r_{i}=1, q_{i}=1} g(\ldots, k) \equiv\left|\rho_{j}(z)\right\rangle_{k}
$$

satisfies the linear equations that follow from Eq. (127),

$$
\hat{M}(z)\left|\rho_{j}(z)\right\rangle=\left|\beta_{j}(z)\right\rangle,
$$

where $\left|\beta_{j}\right\rangle$ is the vector with the components $\left|\beta_{j}\right\rangle_{i}, i=$ $1, \ldots, N_{r}$ defined by

$$
\left|\beta_{j}(z)\right\rangle_{i} \equiv z \kappa_{i \rightarrow j} g(z ; j)\left[1-\delta_{i j}\right]+\kappa_{j \rightarrow j} g(z ; j) \delta_{i j} .
$$

By the same considerations that lead to Eq. (102), we now obtain

$$
\left.\lim _{n \rightarrow \infty} \frac{\partial_{z}^{n} \partial_{r_{j}} g\left(\ldots, i^{\prime}\right)}{\partial_{z}^{n} \partial_{q_{j}} g\left(\ldots, i^{\prime}\right)}\right|_{z=0, r_{i}=1, q_{i}=1}=\left.\frac{\left\langle u^{0} \mid \beta_{j}\right\rangle}{\left\langle u^{0} \mid Q_{j}\right\rangle}\right|_{z=z_{*}},
$$

where, as before, $\left\langle u^{0}\left(z_{*}\right)\right|$ is the unique eigenvector of the matrix $\hat{M}\left(z_{*}\right)$ with eigenvalue 0 . (The normalization of 
$\left\langle u^{0}\right|$ is again irrelevant.) Using the definitions (87) and (131) and the relationship (103), we compute

$$
\begin{aligned}
\left.\frac{\left\langle u^{0} \mid \beta_{j}\right\rangle}{\left\langle u^{0} \mid Q_{j}\right\rangle}\right|_{z=z_{*}} & =\frac{u_{j}^{0}\left(z_{*}\right) \kappa_{j \rightarrow j}+\sum_{i \neq j} u_{i}^{0}\left(z_{*}\right) z_{*} \kappa_{i \rightarrow j}}{\sum_{i \neq j} u_{i}^{0}\left(z_{*}\right) z_{*} \kappa_{i \rightarrow j}} \\
& =\frac{\frac{1}{\nu} g^{\frac{1}{\nu}-1}\left(z_{*} ; j\right)}{\frac{1}{\nu} g^{\frac{1}{\nu}-1}\left(z_{*} ; j\right)-\kappa_{j \rightarrow j}} .
\end{aligned}
$$

The last ratio is always very close to 1 since typically $g\left(z_{*} ; j\right) \ll 1$ while $\kappa_{j \rightarrow j} \approx 1$. In particular, $g\left(z_{*} ; j\right) \ll 1$ for vacua $j$ with low-energy Hubble scale, since for those vacua we may approximate $g\left(z_{*} ; j\right)$ by Eq. (79),

$$
g\left(z_{*} ; j\right) \approx\left[z_{*} \sum_{i \in T} \kappa_{j \rightarrow i}\right]^{\nu} \ll 1 .
$$

Therefore, the RV-regulated ratio $N_{j} / n_{j}$ of the total number of $H$-regions of type $j$ to the total number of bubbles of type $j$ is never large.

Using this result, we can now estimate the RVregulated ratio of $\mathrm{BBs}$ to ordinary observers. The number of ordinary observers per one $H$-region of type $j$ is not precisely known but is presumably at least of order 1 or larger, as long as bubbles of type $j$ are compatible with life. On the other hand, the number of Boltzmann brains per $H$-region, i.e. within a four-volume $H_{j}^{-4}$ of spacetime, is negligibly small. It follows that the abundance of BBs in the RV measure is always negligible relative to the abundance of ordinary observers in the same bubble type.

\section{Derivation of Eq. (78)}

To derive Eq. (78), we need to consider the expansion of a single initial $H$-region during one Hubble time. Within the "inflation in a box" model, an initial $H$-region of type $j$ is split after one Hubble time $\delta t=H_{j}^{-1}$ into $\nu \equiv e^{3}$ statistically independent "daughter" $H$-regions. ${ }^{4}$ Each of the daughter $H$-regions may change its vacuum type from $j$ to $k \neq j(k=1, \ldots, N)$ with probability $\kappa_{j \rightarrow k}$. The quantity $\kappa_{j \rightarrow j}$ was defined for convenience by Eq. (6) to be the probability of not changing the bubble type $j$ during one Hubble time.

The generating function $g$ is defined by Eq. (76),

$$
g\left(z,\left\{q_{i}\right\} ; j\right) \equiv\left\langle z^{n_{\mathrm{tot}}} q_{1}^{n_{1}} \ldots q_{N}^{n_{N}}\right\rangle_{n_{\mathrm{tot}}<\infty ; j}
$$

where $n_{i}$ is the number of bubbles of type $i$, while the notation $\langle\ldots\rangle_{n_{\text {tot }}<\infty ; j}$ stands for a probabilistic average

\footnotetext{
4 To avoid considering a non-integer number $\nu$ of daughter regions, we may temporarily assume that $\nu$ is an integer parameter. At the end of the derivation, we will set $\nu \equiv e^{3} \approx 20.1$ in the final equations.
}

evaluated for the initial $H$-region of type $j$ on the subensemble of finite total number of bubbles $n_{\text {tot }}$. Note that the initial bubble of type $j$ is not counted in $n_{i}$ or $n_{\text {tot }}$. Our goal is to obtain a relationship between $g\left(z,\left\{q_{i}\right\} ; j\right)$ and the generating functions $g\left(z,\left\{q_{i}\right\} ; k\right)$ with $k \neq j$.

To this end, we equate two expressions for the average $\left\langle z^{n_{\mathrm{tot}}} \prod_{i} q_{i}^{n_{i}}\right\rangle_{n_{\mathrm{tot}}<\infty ; j}$. The first expression is the left-hand side of Eq. (135). The second expression is found by considering the $\nu$ daughter $H$-regions evolved out of the initial $H$-region and by using the fact that the same average for a daughter region of type $k$ is equal to $g\left(z,\left\{q_{i}\right\} ; k\right)$. However, two details need to be accounted for: First, the generating functions $g\left(z,\left\{q_{i}\right\} ; k\right)$ evaluated for the daughter regions do not count the daughter bubbles themselves. Second, the type $k$ of each of the daughter regions is a random quantity. Before deriving a general relationship, let us illustrate the procedure using an example.

It is possible that, say, only two of the daughter regions change their type to $k$ while all other daughter regions retain the initial bubble type $j$. Denote temporarily by $p_{k k j \ldots j}$ the probability of this event; binomial combinatorics yields

$$
p_{k k j \ldots j}=\frac{\nu !}{2 !(\nu-2) !} \kappa_{j \rightarrow k}^{2} \kappa_{j \rightarrow j}^{\nu-2} .
$$

Then the average of $z^{n_{\mathrm{tot}}} \prod_{i} q_{i}^{n_{i}}$ receives a contribution

$$
p_{k k j \ldots j} z^{2} q_{k}^{2}\left[g\left(z,\left\{q_{i}\right\} ; k\right)\right]^{2}\left[g\left(z,\left\{q_{i}\right\} ; j\right)\right]^{2-\nu}
$$

from this event. The factor $z^{2}$ describes two additional bubbles that contribute to $n_{\text {tot }}$; the factor $q_{k}^{2}$ accounts for two additional bubbles of type $k$; no factors of $q_{j}$ appear since no additional bubbles of type $j$ are generated. Finally, the powers of $g$ account for all the bubbles generated in the daughter $H$-regions, but these generating functions do not count the daughter $H$-regions themselves. Those daughter $H$-regions are explicitly counted by the extra factors $z^{2} q_{k}^{2}$.

To compute the average, we need to add the contributions from all the possible events of this kind. Since all of the daughter $H$-regions are independent and statistically equivalent, the average $\left\langle z^{n_{\text {tot }}} \prod_{i} q_{i}^{n_{i}}\right\rangle_{n_{\text {tot }}<\infty ; j}$ splits into the product of $\nu$ averages, each evaluated over a single daughter region.

The average over a single daughter region of type $j$ has contribution from transitions to other types $k \neq j$ and a contribution from the event of no transition. Let us first consider a terminal type $k$. With probability $\kappa_{j \rightarrow k}$ a given daughter region becomes a vacuum of type $k$. Thereafter, no more bubbles will be nucleated inside it; the average of $\left\langle z^{n_{\mathrm{tot}}} \prod_{i} q_{i}^{n_{i}}\right\rangle_{n_{\mathrm{tot}}<\infty ; j}$ over that daughter region is simply $z q_{k}$, meaning that there is a total of one bubbles and only one bubble of type $k$. Hence, the contribution of that event to the statistical average is $\kappa_{j \rightarrow k} z q_{k}$.

Now let us consider a nonterminal type $k \neq j$, $k \notin T$. The corresponding contribution to the average is $\kappa_{j \rightarrow k} z q_{k} g\left(z,\left\{q_{i}\right\} ; k\right)$. Since we have defined 
$g\left(z,\left\{q_{i}\right\} ; k\right) \equiv 1$ when $k$ is a terminal bubble type, we may write the contribution as $\kappa_{j \rightarrow k} z q_{k} g\left(z,\left\{q_{i}\right\} ; k\right)$ for both terminal and nonterminal types $k \neq j$.

Finally, we consider the case of $k=j$ (the daughter region retains the original bubble type). Since no new bubbles were nucleated, the contribution to the average is simply $\kappa_{j \rightarrow j} g\left(z,\left\{q_{i}\right\} ; j\right)$ without any factors of $z$ or $q_{k}$.

Putting these ingredients together, we obtain an equation for $g\left(z,\left\{q_{i}\right\} ; j\right)$,

$$
\begin{aligned}
& g\left(z,\left\{q_{i}\right\} ; j\right) \\
& =\left[\sum_{k \neq j} \kappa_{j \rightarrow k} z q_{k} g\left(z,\left\{q_{i}\right\} ; k\right)+\kappa_{j \rightarrow j} g\left(z,\left\{q_{i}\right\} ; j\right)\right]^{\nu} .
\end{aligned}
$$

This is equivalent to Eq. (78). One can also verify that the binomial expansion of Eq. (138) indeed yields all the terms such as the one given in Eq. (137).

We can now analyze the behavior of $g\left(z,\left\{q_{i}\right\} ; j\right)$ in the limit $z \rightarrow 0$. By definition, the generating function $g\left(z,\left\{q_{i}\right\} ; j\right)$ is a power series in $z$ whose coefficient at $z^{n}$ is equal to the probability of the event that a multiverse has exactly $n$ bubbles to the future of the initial bubble $j$. It is clear that this power series starts with the term $z^{\nu}$, corresponding to the probability that the initial bubble $j$ expands exactly into $\nu$ terminal bubbles, signalling the global end of the multiverse. The probability of having fewer than $\nu$ bubbles in the entire multiverse is equal to zero. ${ }^{5}$ The next term of the binomial expansion is of order $z^{2 \nu-1}$ since it is the product of $z^{\nu-1}$ and $g$ itself. Therefore, the small- $z$ behavior of the generating function $g\left(z,\left\{q_{i}\right\} ; j\right)$ must be given by Eq. (79). This condition, together with analyticity in $z$, selects the unique physically relevant solution of Eqs. (78).

\section{E. Eigenvalues of $\hat{M}(z)$}

According to the definition (85), the matrix $\hat{M}(z)$ has positive elements on the diagonal and nonpositive elements off the diagonal. Such a matrix can be rewritten in the form

$$
\hat{M}(z)=\mu \hat{1}-\hat{A}(z)
$$

\footnotetext{
5 The property that there are exactly $\nu$ daughter bubbles is, of course, an artifact of the "inflation in a box" approximation. In the actual multiverse, one has bubbles of spherical shape that can intersect in complicated ways, so a given bubble may end in one, two, or any other number of terminal bubbles. However, we are using the box approximation to obtain results in the limit of very large total number of bubbles, so we disregard the imprecision in the description of multiverses with a very small total number of bubbles. Also, the value of $\nu$ may be considered a variable parameter of the "box" model; the final results will not be overly sensitive to the value of $\nu$.
}

where a constant $\mu>0$ is introduced, the notation $\hat{1}$ stands for an identity matrix, and $\hat{A}(z)$ is a suitable nonnegative matrix, i.e. a matrix with all nonnegative elements. For instance, we may choose $\mu$ as the largest of the diagonal elements of $\hat{M}$. The theory of nonnegative matrices gives powerful results for the eigenvalues of matrices such as $\hat{M}$ and $\hat{A}$ (see e.g. the book [59]). For the present case, the most important are the properties of the algebraically smallest eigenvalue of the matrix $\hat{M}$.

It will be convenient to drop temporarily the argument $z$ since all the results of matrix theory will hold for every fixed $z$. By the Perron-Frobenius theorem (see [59], chapter 9), under the condition of irreducibility ${ }^{6}$ a nonnegative matrix $\hat{A}$ has a unique nondegenerate, real eigenvalue $\alpha_{0}>0$ such that all the eigenvalues of $\hat{A}$ (which may be complex-valued) are located within the circle $|\lambda| \leq \alpha_{0}$ in the complex $\lambda$ plane. This "dominant" eigenvalue $\alpha_{0}$ has a corresponding (right) eigenvector $\left|v^{0}\right\rangle$ that can be chosen with all strictly positive components $v_{i}^{0}>0$. The same property holds for the relevant left eigenvector $\left\langle u^{0}\right|$ (the matrix need not be symmetric, so the left and the right eigenvectors do not, in general, coincide). Therefore it is possible to choose the eigenvectors $\left\langle u^{0}\right|$ and $\left|v^{0}\right\rangle$ such that the normalization $\left\langle u^{0} \mid v^{0}\right\rangle=1$ holds. This normalization will be convenient for further calculations, and so we assume that such eigenvectors have been chosen.

It follows that $\left|v^{0}\right\rangle$ and $\left\langle u^{0}\right|$ are also the right and left eigenvectors of the matrix $\hat{M}$ with the eigenvalue

$$
\lambda_{0} \equiv \mu-\alpha_{0},
$$

while all the other eigenvalues of $\hat{M}$ are located within the circle $|\mu-\lambda| \leq \alpha_{0}$ in the complex $\lambda$ plane. Since $\alpha_{0}>0$, all the other eigenvalues of $\hat{M}$ are strictly to the right (in the complex plane) of the real eigenvalue $\lambda_{0}$. In other words, $\lambda_{0}$ is the eigenvalue of $\hat{M}$ with the algebraically smallest real part.

Restoring now the argument $z$ of the matrix $\hat{M}$, we find that $\hat{M}$ always has a real, nondegenerate eigenvalue $\lambda_{0}(z)$, which is at the same time the eigenvalue with the algebraically smallest real part among all the eigenvalues of $\hat{M}(z)$. We know that $\operatorname{det} \hat{M}(z)>0$ for sufficiently small $z$; hence $\lambda_{0}(z)>0$ for those $z$. Moreover, $\operatorname{det} \hat{M}(z)$ will remain positive as long as $\lambda_{0}(z)>0$, since no other eigenvalue can become negative unless $\lambda_{0}(z)$ first becomes negative. We will now show that $\operatorname{det} \hat{M}(z)$ cannot remain positive for all real $z>0$. It will then follow

\footnotetext{
6 The irreducibility condition means that any two recyclable vacua in the landscape can be connected by a chain of transitions with nonzero nucleation rates. This condition has been discussed in Refs. [23, 24]. If some subset of vacua form a "disconnected island" in the landscape, such that transitions to and from the "island" are forbidden, one can regard the "island" as a separate irreducible landscape and apply the same technique to it. Hence, we consider only irreducible landscapes in this work.
} 
by continuity of $\lambda_{0}(z)$ that there will be a value $z_{*}$ such that $\lambda_{0}(z)>0$ for all $0<z<z_{*}$ but $\lambda_{0}\left(z_{*}\right)=0$.

We will use the property that the inverse matrix $\hat{M}^{-1}(z)$ has all positive elements as long as $\lambda_{0}(z)>0$ (equivalently if $\alpha_{0}<\mu$ ). The derivation of this property is simple:

$$
\hat{M}^{-1}=(\mu \hat{1}-\hat{A})^{-1}=\mu^{-1} \hat{1}+\mu^{-2} \hat{A}+\mu^{-3} \hat{A}^{2}+\ldots
$$

which yields explicitly a matrix with all nonnegative elements. [The matrix-valued series in Eq. (141) converges because all the eigenvalues of $\hat{A}$ are strictly smaller than $\mu$ by absolute value.] Moreover, the irreducibility condition means that some chain of transitions will connect every pair of recyclable vacua; this is equivalent to saying that for any vacua $i, j$ there exists some integer $s$ such that $\hat{A}^{s}$ has a nonzero matrix element $\left(\hat{A}^{s}\right)_{i j}$. Hence, every matrix element of $\hat{M}^{-1}$ is strictly positive as long as $\lambda_{0}(z)>0$.

Further, we can deduce that $g(z ; j)$ is a strictly increasing, real-valued function of $z$ for those $z$ for which $\lambda_{0}(z)>0$. To show this, we consider the vector $\left|\partial_{z} g\right\rangle$ whose components are the $N_{r}$ derivatives $\partial_{z} g(z ; j), j=$ $1, \ldots, N_{r}$. It follows from Eq. (78) that the vector $\left|\partial_{z} g\right\rangle$ satisfies the inhomogeneous equation

$$
\hat{M}(z)\left|\partial_{z} g\right\rangle=|\zeta\rangle,
$$

where we denoted by $|\zeta\rangle$ the vector with the components

$$
\zeta_{k}(z) \equiv \sum_{i \neq k} \kappa_{k \rightarrow i} g(z ; i)
$$

The solution of Eq. (142) is

$$
\left|\partial_{z} g\right\rangle=\hat{M}^{-1}(z)|\zeta(z)\rangle \text {. }
$$

Since all the matrix elements of $\hat{M}^{-1}(z)$ are positive and all the components of $|\zeta\rangle$ are nonnegative as long as $g(z ; i)>0$, it follows that all the components of $\left|\partial_{z} g\right\rangle$ are strictly positive. Equation (79) shows that $g(z ; i)>0$ for sufficiently small $z>0$, and it follows that $g(z ; i)$ will remain positive for all $z>0$ such that $\lambda_{0}(z)>0$. Therefore, $g(z ; i), i=1, \ldots, N_{r}$ are strictly increasing functions of $z$ for all those $z$.

Nevertheless, the functions $g(z ; i)$ are bounded from above. To see this, consider the relationship

$$
\left\langle u^{0}\right| \hat{M}=\lambda_{0}\left\langle u^{0}\right|
$$

written in components as

$$
u_{j}^{0}(z)\left[\frac{1}{\nu} g^{\frac{1}{\nu}-1}(z ; j)-\kappa_{j \rightarrow j}\right]-\sum_{i \neq j} u_{i}^{0}(z) z \kappa_{i \rightarrow j}=\lambda_{0} u_{j}^{0} .
$$

Since all the components $u_{i}^{0}$ are strictly positive (as long as $\lambda_{0}(z)>0$ ), it follows that

$$
\frac{1}{\nu} g^{\frac{1}{\nu}-1}(z ; j)-\kappa_{j \rightarrow j}>0
$$

and hence

$$
g(z ; j)<\left(\nu \kappa_{j \rightarrow j}\right)^{-\frac{\nu}{\nu-1}}<\frac{1}{\nu^{\frac{\nu}{\nu-1}}} \approx \frac{1}{\nu} .
$$

Furthermore, we can show that $\lambda_{0}(z)$ monotonically decreases as $z$ grows. This follows from the perturbation theory formula for nondegenerate eigenvalues, which allows us to express $d \lambda_{0} / d z$ as a matrix product with normalized left and right eigenvectors,

$$
\frac{d \lambda_{0}(z)}{d z}=\left\langle u^{0}(z)\left|\frac{d \hat{M}}{d z}\right| v^{0}(z)\right\rangle \text {. }
$$

As we have just shown, $g(z ; i)$ grows with growing $z$, so $d \hat{M} / d z$ is a matrix with all nonpositive elements. Since the vectors $\left\langle u^{0}\right|$ and $\left|v^{0}\right\rangle$ have strictly positive components while at least some matrix elements of the nonpositive matrix $d \hat{M} / d z$ are strictly negative, we obtain the strict inequality

$$
\frac{d \lambda_{0}(z)}{d z}=\left\langle u^{0}(z)\left|\frac{d \hat{M}}{d z}\right| v^{0}(z)\right\rangle<0
$$

Similarly, we can show that $\operatorname{det} \hat{M}(z)$ monotonically decreases with $z$ :

$$
\frac{d}{d z} \operatorname{det} \hat{M}(z)=(\operatorname{det} \hat{M}(z)) \operatorname{Tr}\left(\hat{M}^{-1} \frac{d \hat{M}}{d z}\right)<0
$$

since it was already found that the matrix $\hat{M}^{-1}(z)$ has all positive elements while $d \hat{M} / d z$ has all nonpositive elements. However, the monotonic decrease alone of $\lambda_{0}(z)$ and of $\operatorname{det} \hat{M}(z)$ is not yet sufficient to establish that the matrix $\hat{M}(z)$ actually becomes singular at some finite $z$.

The results derived so far — the monotonic behavior of $g(z ; j)$ and $\lambda_{0}(z)$, the positivity of the matrix elements of $\hat{M}^{-1}$, the bounds on $g$ - hold for all $z$ for which $\lambda_{0}(z)>0$. Now we will show that $\lambda_{0}(z)$ cannot remain positive for all real $z>0$. We can rewrite Eq. (84) as

$$
z \sum_{i \neq k} \kappa_{k \rightarrow i} g(z ; i)=g^{\frac{1}{\nu}}(z ; k)-\kappa_{k \rightarrow k} g(z ; k) .
$$

Using the property $g(z ; j)>0$ and the bound (148), we obtain (for every $k$ ) an upper bound on $z$,

$$
z=\frac{g^{\frac{1}{\nu}}(z ; k)-\kappa_{k \rightarrow k} g(z ; k)}{\sum_{i \notin T, i \neq k} \kappa_{k \rightarrow i} g(z ; i)+\kappa_{k \rightarrow T}}<\frac{g^{\frac{1}{\nu}}(z ; k)}{\kappa_{k \rightarrow T}}<\frac{\nu^{1-\nu}}{\kappa_{k \rightarrow T}} .
$$

In other words, no real-valued solutions of Eq. (84) exist for larger $z$. Let us then show that an upper bound on $z$ contradicts the assumption that $\lambda_{0}(z)>0$ for all $z$. We know that there exists a real-valued solution branch $g(z ; j)$ near $z=0$ such that $0<g(z ; j)<\infty$ and $0<\partial_{z} g(z ; j)<\infty$ for all those $z>0$ for which this solution branch remains real-valued. Hence, $g(z ; j)$ can be viewed as a solution of a differential equation 
$\partial_{z} g(z ; j)=\left|\partial_{z} g\right\rangle$ with continuous coefficients and everywhere positive right-hand side. The solution of such differential equations, if bounded, will exist for all $z>0$. Indeed, if the solution $g(z ; j)$ existed only up to some $z=z_{1}$, we would have, by assumption, $\lambda_{0}\left(z_{1}\right)>0$ and hence a finite value $g\left(z_{1} ; j\right)>0$ and a finite derivative $\partial_{z} g\left(z_{1} ; j\right)>0$. So the solution $g(z ; j)$ could then be continued further to some $z>z_{1}$. Therefore, the real-valued solution branch $g(z ; j)$ must exist for all $z>0$. This is incompatible with the bound (153).

We conclude that there exists a value $z_{*}>0$ such that $\lambda_{0}\left(z_{*}\right)=0$ but $\lambda_{0}(z)>0$ for all $0<z<z_{*}$. Within the range $0<z<z_{*}$ the functions $g(z ; j)$ grow monotonically but remain bounded by Eq. (148), while $\lambda_{0}(z)$ and $\operatorname{det} \hat{M}(z)$ both decrease monotonically to zero.

\section{F. The root of $\lambda_{0}(z)$}

It remains to establish that $\lambda_{0}(z)$ indeed has the form (89) near $z=z_{*}$. We again restrict our attention to the interval $0<z<z_{*}$ where $\lambda_{0}(z)>0$. For these $z$ we expand $\lambda_{0}(z)$ in Taylor series and express the value $\lambda_{0}\left(z_{*}\right) \equiv 0$ as

$$
0=\lambda_{0}\left(z_{*}\right)=\lambda_{0}(z)+\frac{d \lambda_{0}(z)}{d z}\left(z_{*}-z\right)+O\left[\left(z_{*}-z\right)^{2}\right],
$$

hence

$$
\frac{d \lambda_{0}(z)}{d z}=-\frac{\lambda_{0}(z)}{z_{*}-z}+O\left(z_{*}-z\right) .
$$

We then use Eq. (149) to express $d \lambda_{0} / d z$ in another way,

$$
\begin{aligned}
\frac{d \lambda_{0}(z)}{d z} & =\left\langle u^{0}(z)\left|\frac{d}{d z} \hat{M}(z)\right| v^{0}(z)\right\rangle \\
= & \left\langle u^{0}(z)\left|\left[\sum_{i} \frac{\partial \hat{M}}{\partial g(z ; i)} \partial_{z} g(z ; i)+\partial_{z} \hat{M}(z)\right]\right| v^{0}(z)\right\rangle,
\end{aligned}
$$

where in the second line we interpreted $\hat{M}(z)$ as a function of $N_{r}$ variables $g(z ; i), i=1, \ldots, N_{r}$, and explicitly of $z$, in order to express $d / d z$ through $\partial / \partial g$ and $\partial / \partial z$. Using Eq. (144), we then find

$$
\frac{\lambda_{0}(z)}{z-z_{*}} \approx\left\langle u^{0}(z)\left|\left[\sum_{i, k} \frac{\partial \hat{M}}{\partial g(z ; i)} \hat{M}_{i k}^{-1} \zeta_{k}+\frac{\partial \hat{M}(z)}{\partial z}\right]\right| v^{0}(z)\right\rangle .
$$

Since we are only interested in the qualitative behavior of $\lambda_{0}(z)$ at $z=z_{*}$, we do not need to keep track of the complicated coefficients in Eq. (157). Near $z=z_{*}$ we have, by Eq. (94),

$$
\hat{M}^{-1}(z) \approx \frac{1}{\lambda_{0}(z)}\left|v^{0}\right\rangle\left\langle u^{0}\right|+O(1),
$$

so the dominant singular terms in Eq. (157) near $z=z_{*}$ are

$$
-\frac{\lambda_{0}(z)}{z_{*}-z} \approx \frac{C_{1}}{\lambda_{0}(z)}+O(1)
$$

Therefore we obtain

$$
\lambda_{0}(z)=c_{1} \sqrt{z_{*}-z}+O\left(z_{*}-z\right) .
$$

The positivity of $\lambda_{0}(z)$ for $z<z_{*}$ entails $c_{1}>0$. This concludes the derivation of Eq. (89).

\section{Acknowledgments}

The author thanks Martin Bucher, Jaume Garriga, Andrei Linde, Misao Sasaki, Takahiro Tanaka, Vitaly Vanchurin, and Alex Vilenkin for valuable discussions.
[1] J. Garcia-Bellido, A. D. Linde, and D. A. Linde, Fluctuations of the gravitational constant in the inflationary Brans-Dicke cosmology, Phys. Rev. D50, 730 (1994), astro-ph/9312039.

[2] J. Garcia-Bellido, Jordan-Brans-Dicke stochastic inflation, Nucl. Phys. B423, 221 (1994), astro-ph/9401042.

[3] R. Bousso and J. Polchinski, Quantization of four-form fluxes and dynamical neutralization of the cosmological constant, JHEP 06, 006 (2000), hep-th/0004134.

[4] L. Susskind, The anthropic landscape of string theory (2003), hep-th/0302219.

[5] S. Kachru, R. Kallosh, A. Linde, and S. P. Trivedi, De sitter vacua in string theory, Phys. Rev. D68, 046005 (2003), hep-th/0301240.

[6] M. R. Douglas, The statistics of string / M theory vacua,
JHEP 05, 046 (2003), hep-th/0303194.

[7] J. Garriga and A. Vilenkin, Recycling universe, Phys. Rev. D57, 2230 (1998), astro-ph/9707292.

[8] S. R. Coleman and F. De Luccia, Gravitational effects on and of vacuum decay, Phys. Rev. D21, 3305 (1980).

[9] J. Garriga, A. H. Guth, and A. Vilenkin, Eternal inflation, bubble collisions, and the persistence of memory (2006), hep-th/0612242.

[10] R. Bousso et al., Future Foam (2008), 0807.1947.

[11] A. D. Linde, D. A. Linde, and A. Mezhlumian, From the Big Bang theory to the theory of a stationary universe, Phys. Rev. D49, 1783 (1994), gr-qc/9306035.

[12] A. H. Guth, Inflation and eternal inflation, Phys. Rept. 333, 555 (2000), astro-ph/0002156.

[13] S. Winitzki, Predictions in eternal inflation, Lect. Notes 
Phys. 738, 157 (2008), gr-qc/0612164.

[14] J. Garcia-Bellido and A. D. Linde, Stationarity of inflation and predictions of quantum cosmology, Phys. Rev. D51, 429 (1995), hep-th/9408023.

[15] A. Vilenkin, Predictions from quantum cosmology, Phys. Rev. Lett. 74, 846 (1995), gr-qc/9406010.

[16] A. Vilenkin, Making predictions in eternally inflating universe, Phys. Rev. D52, 3365 (1995), gr-qc/9505031.

[17] A. Aguirre, S. Gratton, and M. C. Johnson, Hurdles for recent measures in eternal inflation (2006), hepth/0611221.

[18] A. Vilenkin, A measure of the multiverse, J. Phys. A40, 6777 (2007), hep-th/0609193.

[19] A. H. Guth, Eternal inflation and its implications, J. Phys. A40, 6811 (2007), hep-th/0702178.

[20] A. Linde, Towards a gauge invariant volume-weighted probability measure for eternal inflation, JCAP 0706, 017 (2007), arXiv:0705.1160 [hep-th].

[21] R. Bousso, Holographic probabilities in eternal inflation, Phys. Rev. Lett. 97, 191302 (2006), hep-th/0605263.

[22] R. Bousso, B. Freivogel, and I.-S. Yang, Eternal inflation: The inside story (2006), hep-th/0606114.

[23] J. Garriga and A. Vilenkin, Holographic multiverse (2008), 0809.4257.

[24] J. Garriga, D. Schwartz-Perlov, A. Vilenkin, and S. Winitzki, Probabilities in the inflationary multiverse, JCAP 0601, 017 (2006), hep-th/0509184.

[25] R. Easther, E. A. Lim, and M. R. Martin, Counting pockets with world lines in eternal inflation, JCAP 0603, 016 (2006), astro-ph/0511233.

[26] V. Vanchurin and A. Vilenkin, Eternal observers and bubble abundances in the landscape, Phys. Rev. D74, 043520 (2006), hep-th/0605015.

[27] T. Clifton, S. Shenker, and N. Sivanandam, Volumeweighted measures of eternal inflation in the Bousso-Polchinski landscape, JHEP 09, 034 (2007), arXiv:0706.3201 [hep-th].

[28] A. Aguirre, S. Gratton, and M. C. Johnson, Measures on transitions for cosmology from eternal inflation, Phys. Rev. Lett. 98, 131301 (2007), hep-th/0612195.

[29] A. Linde, Sinks in the landscape, Boltzmann brains, and the cosmological constant oroblem, JCAP 0701, 022 (2007), hep-th/0611043.

[30] A. De Simone, A. H. Guth, M. P. Salem, and A. Vilenkin, Predicting the cosmological constant with the scale-factor cutoff measure (2008), 0805.2173.

[31] R. Bousso, B. Freivogel, and I.-S. Yang, Properties of the scale factor measure (2008), 0808.3770.

[32] A. De Simone et al., Boltzmann brains and the scalefactor cutoff measure of the multiverse (2008), 0808.3778.

[33] S. Winitzki and A. Vilenkin, Uncertainties of predictions in models of eternal inflation, Phys. Rev. D53, 4298 (1996), gr-qc/9510054.

[34] A. D. Linde and A. Mezhlumian, On regularization scheme dependence of predictions in inflationary cosmology, Phys. Rev. D53, 4267 (1996), gr-qc/9511058.

[35] A. D. Linde, D. A. Linde, and A. Mezhlumian, Do we live in the center of the world?, Phys. Lett. B345, 203 (1995), hep-th/9411111.

[36] A. Vilenkin, Unambiguous probabilities in an eternally inflating universe, Phys. Rev. Lett. 81, 5501 (1998), hepth/9806185.

[37] L. Dyson, M. Kleban, and L. Susskind, Disturbing implications of a cosmological constant, JHEP 10, 011 (2002), hep-th/0208013.

[38] A. Albrecht and L. Sorbo, Can the universe afford inflation?, Phys. Rev. D70, 063528 (2004), hep-th/0405270.

[39] D. N. Page, Is our universe likely to decay within 20 billion years? (2006), hep-th/0610079.

[40] A. Vilenkin, Freak observers and the measure of the multiverse, JHEP 01, 092 (2007), hep-th/0611271.

[41] D. N. Page, Return of the Boltzmann brains (2006), hepth/0611158.

[42] R. Bousso, B. Freivogel, and I.-S. Yang, Boltzmann babies in the proper time measure (2007), arXiv:0712.3324 [hepth].

[43] J. R. Gott III, Boltzmann brains-I'd rather see than be one (2008), arXiv:0802.0233 [gr-qc].

[44] D. Schwartz-Perlov and A. Vilenkin, Probabilities in the Bousso-Polchinski multiverse, JCAP 0606, 010 (2006), hep-th/0601162.

[45] R. Bousso and I.-S. Yang, Landscape predictions from cosmological vacuum selection, Phys. Rev. D75, 123520 (2007), hep-th/0703206.

[46] R. Bousso, R. Harnik, G. D. Kribs, and G. Perez, Predicting the cosmological constant from the causal entropic principle, Phys. Rev. D76, 043513 (2007), hepth/0702115.

[47] D. Schwartz-Perlov, Probabilities in the Arkani-HamedDimopolous-Kachru landscape, J. Phys. A40, 7363 (2007), hep-th/0611237.

[48] K. D. Olum and D. Schwartz-Perlov, Anthropic prediction in a large toy landscape, JCAP 0710, 010 (2007), arXiv:0705.2562 [hep-th].

[49] D. Schwartz-Perlov, Anthropic prediction for a large multi-jump landscape (2008), 0805.3549.

[50] S. Winitzki, A volume-weighted measure for eternal inflation, Phys. Rev. D78, 043501 (2008), 0803.1300.

[51] S. Winitzki, Reheating-volume measure for random-walk inflation, Phys. Rev. D78, 063517 (2008), 0805.3940.

[52] M. Aryal and A. Vilenkin, The fractal dimension of inflationary universe, Phys. Lett. B199, 351 (1987).

[53] S. Winitzki, On time-reparametrization invariance in eternal inflation, Phys. Rev. D71, 123507 (2005), grqc/0504084.

[54] A. Aguirre, T. Banks, and M. Johnson, Regulating eternal inflation. II: The great divide, JHEP 08, 065 (2006), hepth/0603107.

[55] D. I. Podolsky, J. Majumder, and N. Jokela, Disorder on the landscape, JCAP 0805, 024 (2008), 0804.2263.

[56] M. C. Johnson and M. Larfors, Field dynamics and tunneling in a flux landscape (2008), 0805.3705.

[57] B. Freivogel and M. Lippert, Evidence for a bound on the lifetime of de Sitter space (2008), 0807.1104.

[58] M. C. Johnson and M. Larfors, An obstacle to populating the string theory landscape (2008), 0809.2604.

[59] P. Lancaster, Theory of matrices (Academic Press, New York, 1969). 